\title{
A Comparative Study of Different Control Structures for Flight Control with New Results
}

\author{
Espen Oland, Raymond Kristiansen and Jan Tommy Gravdahl
}

\begin{abstract}
This paper presents several different control structures that facilitates flight control and does a comparison between them. Specifically, the paper considers command-filtered backstepping, nonlinear-dynamic inversion (NDI) and a new decoupled approach that decouples the rotational and translational dynamics by estimating the higher order derivatives of the angle of attack and sideslip angle. The latter is also augmented by exploiting a feedback of the control deficiency resulting in improved performance. A series of simulations are performed to gauge the performance of the different controllers, showing the performance in the case of sensor noise, when performing aggressive maneuvers, when exposed to wind disturbances, as well as when there are model imperfections. The main finding is that all control structures work well for flight control, but that the new decoupled method is able to improve the performance. A major reason for the improvement is that the decoupling method alleviates the tuning of the control gains, thus allowing for faster response through suitable gains.
\end{abstract}

Index Terms-Aircraft, backstepping, command-filtered backstepping, decoupled control, flight control, nonlinear dynamic inversion, pseudo-control hedging, quaternions.

\section{INTRODUCTION}

$\mathbf{T}$ HE problem of flight control for aircraft can be defined as that of pointing the wind frame in a desired direction and move with a desired positive airspeed. There is a multitude of methods that solves this problem such as kinematic controllers [1], gain scheduling [2], [3], Nonlinear Dynamic Inversion (NDI) [4], [5], [6], regular backstepping [7], [8] and commandfiltered backstepping [9], [10]. Kinematic controllers ignore the nonlinear aerodynamics and controls the aircraft at a kinematic level, while gain scheduling uses a number of linear controllers that provide satisfactory performance at different operating points and switches between them to control the aircraft.

Nonlinear dynamic inversion calculates a desired angular acceleration and inverts the dynamics to find the desired deflection angles that achieves the desired angular acceleration and uses that for control. Specifically, it uses an inner-outer loop structure through time-scale separation, where the deflection angles and angular velocities are considered constant in the outer loop, allowing a desired angular velocity to be

Manuscript received June 25, 2017; revised June 20, 2018.

E. Oland is with the Department of Electrical Engineering at UiT The Arctic University of Norway, Nordland, 8514 Narvik, Norway, e-mail: espen.oland@uit.no.

R. Kristiansen is with the Department of Electrical Engineering at UiT The Arctic University of Norway, Nordland, 8514 Narvik, Norway, e-mail: raymond.kristiansen@uit.no.

J. T. Gravdahl is with the Department of Engineering Cybernetics at Norwegian University of Science and Technology, NO7491 Trondheim, Norway, e-mail: jan.tommy.gravdahl@ntnu.no. calculated. The inner loop then calculates the desired angular acceleration enabling the dynamics to be inverted. Time-scale separation is achieved through gain selection, where the outer loop commonly has a low gain, while the inner loop has a high gain. This puts limitations to the available gains for controlling aircraft through NDI, and requires some tuning to find the best set of gains.

Command-filtered backstepping for flight control has recently received much attention. The method uses commandfilters with magnitude and rate saturation to calculate the command derivatives, thereby enabling trackable commands for the next step in the backstepping process. Specifically, it consists of an outer loop, a middle loop and an inner loop. The outer loop calculates the desired thrust, desired angle of attack and desired bank angle, the middle loop calculates the desired angular velocities, while the inner loop calculates the desired deflection angles that make the aircraft track a desired heading, flight path and bank angle. With three different loops that must be time-scale separated, much effort is required to properly tune the command filters and the different control loops.

In addition to these methods, a method that decouples the rotational and translational dynamics has recently been proposed [11]. The method estimates the higher order derivatives of the angle of attack and sideslip angle, something that allows the rotational and translational dynamics to become decoupled. This allows a rotational controller to be designed first, followed by a speed controller, where the gains can be chosen almost arbitrarily. The work shows good results, but it has never before been benchmarked relative to the state of the art within flight control. Specifically, the method takes basis in the quaternion error between the wind frame and the desired frame consisting of: a desired quaternion, the quaternion representing the orientation of the aircraft and a quaternion representing the rotation from the wind frame to the body frame. To find the error dynamics, the quaternion error must be differentiated twice, resulting in the attitude dynamics of the aircraft, a desired angular acceleration, as well as an angular acceleration due to the wind frame. Since the latter angular acceleration cannot be measured directly, the decoupled method approximates it using a linear filter driven by the angle of attack and sideslip angle and uses its input as part of the control law. After finding the rotational controller, it is straight forward to find a speed controller that makes the airspeed track a desired speed profile.

This paper compares the NDI, the command-filtered backstepping approach and the decoupled method, discusses the results and highlights some of the advantages and disadvantages 
with the different methods. Additionally, it presents a new approach based on pseudo-control hedging [12] that allows a reference trajectory to be designed that increases the control authority of the aircraft and allows for improved performance.

The paper is structured as follows: Section $\amalg$ presents the notation, reference frames and modeling of aircraft, then the main body of the paper follows through Section III which presents the different control structures in detail showing how each of them can achieve the control objective. Section IV then presents a comparison between the methods through four different simulation scenarios: First, the methods are compared by considering a maneuver of changing the orientation by $180^{\circ}$ and achieving a desired airspeed; then a study is performed to see the impact of sensor noise; then a more aggressive maneuver is performed in a more realistic environment using the Dryden gust model and wind shear model; and lastly, a comparison between the methods on their ability to cope with modeling imperfections is performed. The paper is then wrapped up with a discussion and conclusion through Sections $\mathrm{V}$ and $\mathrm{VI}$.

\section{MODELING}

\section{A. Notation}

The time derivative is denoted $\dot{x}=\frac{d}{d t} x$, vectors are bold small letters, and matrices are bold capital letters. The superscript of a vector denotes its reference frame where $d$ denotes the desired frame, $b$ denotes the body frame, $s$ denotes the stability frame, $w$ denotes the wind frame, $v$ denotes the velocity frame, $n$ denotes the North East Down (NED) frame, and $r$ denotes a reference trajectory frame, which is used in this paper to account for actuator saturation. The rotation matrix that rotates a vector from frame $a$ to frame $c$ is denoted $\mathbf{R}_{a}^{c} \in \mathcal{S O}(3)=\left\{\mathbf{R}^{\top} \mathbf{R}=\mathbf{I} \in \mathbb{R}^{3}, \operatorname{det}(\mathbf{R})=1\right\}$ where $\mathbf{I}$ is the identity matrix. The time derivative of the rotation matrix is given as $\dot{\mathbf{R}}_{a}^{c}=\mathbf{R}_{a}^{c} \mathbf{S}\left(\boldsymbol{\omega}_{c, a}^{a}\right)$ where $\mathbf{S}(\cdot)$ is the cross-product operator and $\boldsymbol{\omega}_{c, a}^{a}$ represents the angular velocity of frame $a$ relative to frame $c$ referenced in frame $a$. The cross product operator can be written for a vector $\mathbf{v}=\left[\begin{array}{lll}v_{1} & v_{2} & v_{3}\end{array}\right]^{\top}$ as

$$
\mathbf{S}(\mathbf{v})=\left[\begin{array}{ccc}
0 & -v_{3} & v_{2} \\
v_{3} & 0 & -v_{1} \\
-v_{2} & v_{1} & 0
\end{array}\right] \text {. }
$$

The quaternion representing the rotation of a vector from frame $a$ to frame $c$ is denoted as $\mathbf{q}_{c, a} \in \mathcal{S}^{3}=\left\{\mathbf{q} \in \mathbb{R}^{4}\right.$ : $\left.\mathbf{q}^{\top} \mathbf{q}=1\right\}$ and where the quaternion error between two frames can be written as the composite quaternion $\mathbf{q}_{c, a}=\mathbf{q}_{c, e} \otimes \mathbf{q}_{e, a}$, where $e$ is an intermediate frame. The notation of Euler angles are defined similarly as for quaternions, where $\boldsymbol{\Theta}_{c, a} \in \mathbb{R}^{3}$ represents the orientation of frame $a$ relative to frame $c$.

\section{B. Reference Frames}

Several reference frames are required to fully describe the aircraft dynamics. Fig. 1 shows the relationships between the different frames, which can be formally defined as follows:

North East Down: The NED frame has the $\mathrm{x}^{n}$ axis pointing towards the north pole, $\mathbf{y}^{n}$ points East and $\mathbf{z}^{n}$ points down

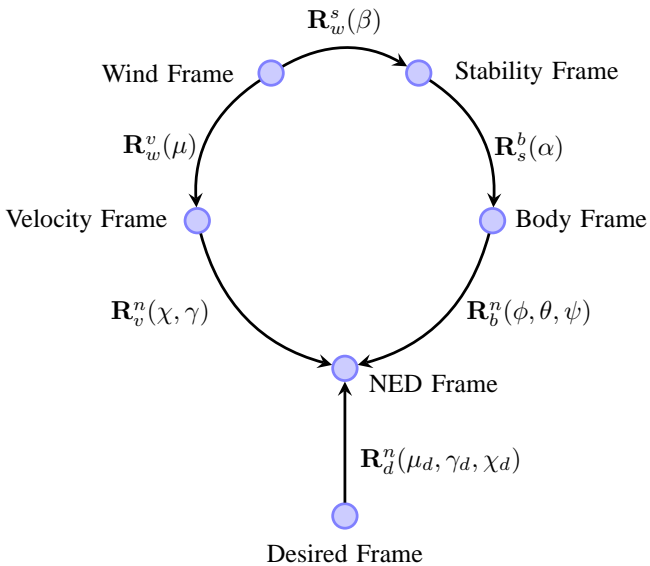

Fig. 1. The rotation wheel describing the different reference frames required for flight control (Inspired by [14]).

towards the center of the Earth. This reference frame is treated as an inertial frame (also known as flat-Earth approximation).

Body: The body frame is fixed to the center of mass of the aircraft where the $\mathrm{x}^{b}$ axis goes through the fuselage towards the nose of the aircraft, $\mathbf{y}^{b}$ goes through the right wing, and $\mathbf{z}^{b}$ completes the right-handed orthonormal system.

Stability: The stability frame is initially aligned with the body frame and is found by rotating by the angle of attack $(\alpha)$ around the $\mathbf{y}^{b}$ axis as a counter-clockwise rotation.

Wind: The wind frame is initially aligned with the stability frame, and is found by rotating by the sideslip angle around the $\mathbf{z}^{s}$ axis. In the wind frame, the airspeed is aligned along the $\mathbf{x}^{w}$ axis.

Velocity: The velocity frame describes the direction that the aircraft is moving and can be described using the heading $(\chi)$ and flight path angle $(\gamma)$ which are described relative to the NED frame. In the velocity frame the airspeed is also aligned along the $\mathrm{x}^{v}$ axis, but the wind frame and velocity frame are not the same, and are related through the bank angle $\mu$.

Desired: The desired frame can be described arbitrarily depending on the objective. In the recent paper [13], it is shown how to design desired orientations to facilitate waypoint tracking, collision avoidance, ground avoidance, wind compensation and how to fuse them together using the subsumption method.

\section{Flight Dynamics}

The translational dynamics for an aircraft can be written as (cf. [15])

$$
\begin{aligned}
\dot{\mathbf{p}}^{n} & =\mathbf{R}_{b}^{n} \mathbf{v}^{b} \\
\mathbf{v}_{r}^{b} & =\mathbf{v}^{b}-\mathbf{R}_{n}^{b} \mathbf{w}^{n} \\
\dot{\mathbf{v}}_{r}^{b} & =\frac{1}{m} \mathbf{f}_{\text {thrust }}^{b}+\frac{1}{m} \mathbf{R}_{w}^{b} \mathbf{f}_{\text {aero }}^{w}+\mathbf{R}_{n}^{b} \mathbf{f}_{g}^{n}-\mathbf{S}\left(\boldsymbol{\omega}_{n, b}^{b}\right) \mathbf{v}_{r}^{b}
\end{aligned}
$$

where $\mathbf{p}^{n}$ is the position vector, $\mathbf{v}^{b}$ is the velocity vector relative to the ground, $\mathbf{v}_{r}^{b}:=\left[\begin{array}{lll}u & v & w\end{array}\right]^{\top}$ is the velocity vector relative the surrounding air, $\mathbf{w}^{n}$ is the wind vector, $m$ is the mass, $\mathbf{f}_{\text {thrust }}^{b}=\left[\begin{array}{ccc}T & 0 & 0\end{array}\right]^{\top}$ is the thrust vector with 
$T \geq 0$ as the total thrust, $\mathbf{f}_{g}^{n}=\left[\begin{array}{lll}0 & 0 & g\end{array}\right]^{\top}$ is the gravity vector where $g=9.81 \mathrm{~m} / \mathrm{s}^{2}$ is the acceleration due to gravity, $\boldsymbol{\omega}_{n, b}^{b}:=\left[\begin{array}{lll}p & q & r\end{array}\right]^{\top}$ is the angular velocity of the body frame relative to the NED frame referenced in the body frame. The aerodynamic force vector is commonly represented in the wind frame as

$$
\begin{aligned}
\mathbf{f}_{\text {aero }}^{w}= & \frac{1}{2} \rho S V_{a}^{2}\left[\begin{array}{lll}
-C_{D} & C_{Y} & -C_{L}
\end{array}\right]^{\top} \\
C_{D}= & C_{D_{0}}+C_{D_{\alpha}} \alpha+\frac{\bar{c}}{2 V_{a}} C_{D_{q}} q+C_{D_{\delta_{e}}} \delta_{e} \\
C_{Y}= & C_{Y_{0}}+C_{Y_{\beta}} \beta+\frac{b}{2 V_{a}} C_{Y_{p}} p+\frac{b}{2 V_{a}} C_{Y_{r}} r \\
& +C_{Y_{\delta_{a}}} \delta_{a}+C_{Y_{\delta_{r}}} \delta_{r} \\
C_{L}= & C_{L_{0}}+C_{L_{\alpha}} \alpha+\frac{\bar{c}}{2 V_{a}} C_{L_{q}} q+C_{L_{\delta_{e}}} \delta_{e}
\end{aligned}
$$

where $C_{(\cdot)}$ represent aerodynamic coefficients, $\rho$ is the air density, $S$ is the surface area of the wings, $b$ is the wing span, $\bar{c}$ is the mean aerodynamic chord, $V_{a}=\left\|\mathbf{v}_{r}^{b}\right\|=\sqrt{\left(\mathbf{v}_{r}^{b}\right)^{\top} \mathbf{v}_{r}^{b}}$ is the airspeed of the aircraft and $\delta_{a}, \delta_{e}, \delta_{r}$ represent the deflection angles of the ailerons, elevator and rudder respectively. The angle of attack is found as $\alpha=\sin ^{-1}\left(\frac{w}{u}\right)$ and the sideslip angle is found as $\beta=\sin ^{-1}\left(\frac{v}{V_{a}}\right)$. The time derivative of the airspeed can be found as

$$
\dot{V}_{a}=\frac{d}{d t}\left\|\mathbf{v}_{r}^{b}\right\|=\frac{u}{m V_{a}} T+\frac{\left(\mathbf{v}_{r}^{b}\right)^{\top}}{V_{a}}\left(\frac{1}{m} \mathbf{R}_{w}^{b} \mathbf{f}_{\text {aero }}^{w}+\mathbf{R}_{n}^{b} \mathbf{f}_{g}^{n}\right)
$$

allowing relatively simple speed controllers to be designed.

The rotational dynamics can be written using Euler angles or quaternions as ( $c f$. [15], [16], [17])

$$
\begin{aligned}
\dot{\boldsymbol{\Theta}}_{n, b} & =\mathbf{T}\left(\boldsymbol{\Theta}_{n, b}\right) \boldsymbol{\omega}_{n, b}^{b} \\
\dot{\mathbf{q}}_{n, b} & =\frac{1}{2} \mathbf{q}_{n, b} \otimes\left[\begin{array}{c}
0 \\
\boldsymbol{\omega}_{n, b}^{b}
\end{array}\right]=\frac{1}{2} \mathbf{T}\left(\mathbf{q}_{n, b}\right)\left[\begin{array}{c}
0 \\
\boldsymbol{\omega}_{n, b}^{b}
\end{array}\right] \\
\mathbf{J} \dot{\boldsymbol{\omega}}_{n, b}^{b} & =-\mathbf{S}\left(\boldsymbol{\omega}_{n, b}^{b}\right) \mathbf{J} \boldsymbol{\omega}_{n, b}^{b}+\mathbf{f}(\mathbf{x})-\mathbf{D}(\mathbf{x}) \boldsymbol{\omega}_{n, b}^{b}+\mathbf{G}(\mathbf{x}) \mathbf{u}
\end{aligned}
$$

where $\boldsymbol{\Theta}_{n, b}=\left[\begin{array}{lll}\phi & \theta & \psi\end{array}\right]^{\top}$ is the vector of Euler angles representing the roll, pitch and yaw angles with

$$
\mathbf{T}(\Theta)=\left[\begin{array}{ccc}
1 & \sin (\phi) \tan (\theta) & \cos (\phi) \tan (\theta) \\
0 & \cos (\phi) & -\sin (\phi) \\
0 & \frac{\sin (\phi)}{\cos (\theta)} & \frac{\cos (\phi)}{\cos (\theta)}
\end{array}\right],
$$

while the quaternion is defined as $\mathbf{q}_{n, b}=\left[\begin{array}{ll}\eta_{n, b} & \boldsymbol{\epsilon}_{n, b}^{\top}\end{array}\right]^{\top}$ with

$$
\mathbf{T}\left(\mathbf{q}_{n, b}\right)=\left[\begin{array}{cc}
\eta_{n, b} & -\boldsymbol{\epsilon}_{n, b}^{\top} \\
\boldsymbol{\epsilon}_{n, b} & \eta_{n, b} \mathbf{I}+\mathbf{S}\left(\boldsymbol{\epsilon}_{n, b}\right)
\end{array}\right] .
$$

The inertia matrix is given as

$$
\mathbf{J}=\left[\begin{array}{ccc}
J_{x x} & 0 & -J_{x z} \\
0 & J_{y y} & 0 \\
-J_{x z} & 0 & J_{z z}
\end{array}\right]
$$

where $J_{x x}, J_{y y}, J_{z z}, J_{x z}$ represent constant positive inertia components. The aerodynamic vector $\mathbf{f}(\mathbf{x})$ is given as

$$
\mathbf{f}(\mathbf{x})=\frac{1}{2} \rho S V_{a}^{2}\left[\begin{array}{c}
b\left(C_{l_{0}}+C_{l_{\beta}} \beta\right) \\
\bar{c}\left(C_{m_{0}}+C_{m_{\alpha}} \alpha\right) \\
b\left(C_{n_{0}}+C_{n_{\beta}} \beta\right)
\end{array}\right]
$$

and the damping matrix as

$$
\mathbf{D}(\mathbf{x})=-\frac{1}{2} \rho S V_{a}^{2}\left[\begin{array}{ccc}
\frac{b^{2}}{2 V_{a}} C_{l_{p}} & 0 & \frac{b^{2}}{2 V_{a}} C_{l_{r}} \\
0 & \frac{\bar{c}^{2}}{2 V_{a}} C_{m_{q}} & 0 \\
\frac{b^{2}}{2 V_{a}} C_{n_{p}} & 0 & \frac{b^{2}}{2 V_{a}} C_{n_{r}}
\end{array}\right] .
$$

Note that $\mathbf{D}(\mathbf{x})$ is positive definite for all $V_{a}>0$, which together with the limits in actuation makes the angular velocity bounded. The control vector is defined as $\mathbf{u}=\left[\begin{array}{lll}\delta_{a} & \delta_{e} & \delta_{r}\end{array}\right]^{\top}$ and the control effectiveness matrix as

$$
\mathbf{G}(\mathbf{x})=\frac{1}{2} \rho S V_{a}^{2}\left[\begin{array}{ccc}
b C_{l_{\delta_{a}}} & 0 & b C_{l_{\delta_{r}}} \\
0 & \bar{c} C_{m_{\delta_{e}}} & 0 \\
b C_{n_{\delta_{a}}} & 0 & b C_{n_{\delta_{r}}}
\end{array}\right]
$$

which is invertible as long as $C_{m_{\delta_{e}}}\left(C_{l_{\delta_{a}}} C_{n_{\delta_{r}}}-C_{l_{\delta_{r}}} C_{n_{\delta_{a}}}\right) \neq$ 0 . The rotation matrix can now be constructed using either quaternions as $\mathbf{R}_{b}^{n}=\mathbf{I}+2 \eta_{n, b} \mathbf{S}\left(\boldsymbol{\epsilon}_{n, b}\right)+2 \mathbf{S}^{2}\left(\boldsymbol{\epsilon}_{n, b}\right)$ or using Euler angles as shown in (19) (cf. [18]). In the following, both methods will be employed depending on the control structure. Note that even though Euler angle kinematics has singularities at $\theta= \pm \frac{\pi}{2}$, there are ways to deal with that, something that is outside the scope of this paper.

\section{Control Structures}

This paper examines three different control structures. First it considers command-filtered backstepping using mixed dynamics where scalar equations from both the rotational and translational dynamics are combined. By mixing equations like this, the good properties of the rotation matrix and skewsymmetric matrices are lost, and the dynamics become singular at certain angles. The second method that is examined is the well-known Nonlinear-Dynamic Inversion (NDI), which dates back to the 1980's. While it has mainly been used to track desired angle of attack and sideslip angles, which are not defined relative to an inertial frame, the method is changed to facilitate tracking of desired heading, flight path and bank angles. The third approach that is addressed, is a new decoupled method where the higher order derivatives of the angle of attack and sideslip angle can be approximated using a simple filter, something that decouples the rotational and translational systems, allowing a rotational controller to be designed first followed by a translational controller.

Fig. 2 shows the structure of the command-filtered backstepping controller, Fig. 3 shows the structure of the NDI controller, and Fig. 4 shows the structure of the decoupled method with a reference. The main differences between the

$$
\mathbf{R}_{b}^{n}=\left[\begin{array}{ccc}
\cos (\psi) \cos (\theta) & -\sin (\psi) \cos (\phi)+\cos (\psi) \sin (\theta) \sin (\phi) & \sin (\psi) \sin (\phi)+\cos (\psi) \cos (\phi) \sin (\theta) \\
\sin (\psi) \cos (\theta) & \cos (\psi) \cos (\phi)+\sin (\phi) \sin (\theta) \sin (\psi) & -\cos (\psi) \sin (\phi)+\sin (\theta) \sin (\psi) \cos (\phi) \\
-\sin (\theta) & \cos (\theta) \sin (\phi) & \cos (\theta) \cos (\phi)
\end{array}\right]
$$


methods are as follows: The command-filtered backstepping approach comprises three time-scale separated loops, where the outputs are filtered to provide trackable commands for the next loop. Especially, note that both the thrust and deflection angles are used in the outer and middle loops to calculate the desired commands, requiring the previous values to calculate the new commands. In the NDI approach, the desired Euler angles are compensated for the angle of attack and sideslip angle to enable the NDI controller to track desired bank, flight path and heading angles. The NDI comprises two main loops, a slow outer loop and a fast inner loop. The gains for both the command-filtered backstepping and NDI controllers must be chosen carefully to maintain time-scale separation. The last method is the decoupled approach. Given desired quaternion, angular velocity and acceleration, the required deflection angles can be found to make the aircraft track the desired orientation. Furthermore, the computed deflection angles can then be used as part of the speed controller to track a desired speed profile. The filter uses measurements of the angle of attack and sideslip angle to decouple the rotational and translational dynamics. To further improve the performance of the approach, a reference generator is used to account for actuator saturation. The control structure for the decoupled method without reference is similar to Fig. 4, but without the reference block.

The control problem can now be formally defined. Let $\tilde{V}=V_{a}-V_{d}$ denote the speed error with $V_{d}, \dot{V}_{d} \in \mathcal{L}_{\infty}$ as desired airspeed and acceleration, and let the tracking errors of the heading, flight path and bank angle be defined respectively as $\tilde{\chi}=\chi-\chi_{d}, \tilde{\gamma}=\gamma-\gamma_{d}$ and $\tilde{\mu}=\mu-\mu_{d}$, where subscript $d$ denotes bounded desired angles with bounded derivatives, then the control objective is to make $(\tilde{V}, \tilde{\chi}, \tilde{\gamma}, \tilde{\mu}) \rightarrow(0,0,0,0)$. This will be achieved using the three different control structures.

\section{A. Command-Filtered Backstepping}

The wind frame can be related to the NED frame through the flight path, heading and bank angle. The velocity vector (4) can be rotated to the velocity frame and differentiated, allowing the speed and angular rates to be found as described in [19, p. 65]. Let $\mathbf{x}_{1}:=\left[\begin{array}{lll}\chi & \gamma & V_{a}\end{array}\right]^{\top}, \mathbf{x}_{2}:=\left[\begin{array}{lll}\mu & \alpha & \beta\end{array}\right]^{\top}$ and $\mathbf{x}_{3}:=\boldsymbol{\omega}_{n, b}^{b}$, then the dynamics can be written as

$$
\begin{aligned}
\dot{\mathbf{x}}_{1} & =\mathbf{f}_{1}\left(\mathbf{x}_{1}, \mathbf{x}_{2}, \mathbf{x}_{3}, T, \mathbf{u}\right)+\mathbf{g}_{1}\left(\mathbf{x}_{1}, \mathbf{x}_{2}, \mathbf{x}_{3}, T, \mathbf{u}\right) \\
\dot{\mathbf{x}}_{2} & =\mathbf{f}_{2}\left(\mathbf{x}_{1}, \mathbf{x}_{2}, \mathbf{x}_{3}, T, \mathbf{u}\right)+\mathbf{G}_{2}\left(\mathbf{x}_{2}\right) \mathbf{x}_{3} \\
\mathbf{J} \dot{\mathbf{x}}_{3} & =\mathbf{f}_{3}\left(\mathbf{x}_{1}, \mathbf{x}_{2}, \mathbf{x}_{3}\right)+\mathbf{G}(\mathbf{x}) \mathbf{u}
\end{aligned}
$$

where $\mathbf{f}_{3}\left(\mathbf{x}_{1}, \mathbf{x}_{2}, \mathbf{x}_{3}\right)=-\mathbf{S}\left(\mathbf{x}_{3}\right) \mathbf{J} \mathbf{x}_{3}+\mathbf{f}(\mathbf{x})-\mathbf{D}(\mathbf{x}) \mathbf{x}_{3}$ and where the other vectors and matrices are described in (23)(26). For this system, the sideslip angle will always be driven to zero, to avoid the singularity of the dynamics when $\beta=$ $\pm \frac{\pi}{2}$. Using backstepping, the state $\mathbf{x}_{1}$ can be driven to desired values using the bank angle, angle of attack and thrust. The bank angle, angle of attack and sideslip can then be driven to desired values using the angular velocities, which again can be controlled using the deflection angles. Hence, under certain assumptions the system is controllable ( $c f$. [10]). Especially note that the angle of attack and bank angle do not appear affine in the $\mathbf{x}_{1}$-system, such that stabilizing that system requires special care. Furthermore, the translational aerodynamics are function of the angular velocity and deflection angles, which therefore must be assumed to be constant (or slowly changing) in the $\mathbf{x}_{1}$-system. This puts strict limitations to the available gains as they must enforce time-scale separation.

This system has been treated in the literature using command-filtered backstepping in works such as [9] and [10], where the system is divided into three different systems that are time-scale separated. The arguments of some of the functions are omitted for ease of notation. In the following subscript $d$ will denote desired states, and the main objective is to make $\mathbf{x}_{1} \rightarrow \mathbf{x}_{1, d}$. First the vector $\mathbf{g}_{1}(\cdot)$ is treated as a control signal to stabilize the outer loop, which can be achieved by the control law

$$
\mathbf{g}_{1}(\cdot)=\dot{\mathbf{x}}_{1, d}-\mathbf{f}_{1}(\cdot)-\mathbf{K}_{1}\left(\mathbf{x}_{1}-\mathbf{x}_{1, d}\right)
$$

where $\mathbf{K}_{1}$ is a positive definite gain matrix. By properly choosing the desired trust $(T)$, bank angle $(\mu)$ and angle

$$
\begin{aligned}
& \mathbf{f}_{1}\left(\mathbf{x}_{1}, \mathbf{x}_{2}, \mathbf{x}_{3}, T, \mathbf{u}\right)=\left[\begin{array}{c}
\frac{1}{m V_{a} \cos (\gamma)}(D \sin (\beta) \cos (\mu)+Y \cos (\mu) \cos (\beta)-T \cos (\mu) \sin (\beta) \cos (\alpha) \\
\frac{1}{m V_{a}}(-D \sin (\beta) \sin (\mu)-Y \sin (\mu) \cos (\beta)+T \sin (\mu) \sin (\beta) \cos (\alpha))-\frac{g}{V_{a}} \cos (\gamma) \\
\frac{1}{m}(-D \cos (\beta)+Y \sin (\beta))-g \sin (\gamma)
\end{array}\right] \\
& \mathbf{g}_{1}\left(\mathbf{x}_{1}, \mathbf{x}_{2}, \mathbf{x}_{3}, T, \mathbf{u}\right)=\left[\begin{array}{c}
\frac{1}{m V_{a} \cos (\gamma)}(L+T \sin (\alpha)) \sin (\mu) \\
\frac{1}{m V_{a}}(L+T \sin (\alpha)) \cos (\mu) \\
\frac{1}{m} T \cos (\beta) \cos (\alpha)
\end{array}\right] \\
& \mathbf{f}_{2}\left(\mathbf{x}_{1}, \mathbf{x}_{2}, \mathbf{x}_{3}, T, \mathbf{u}\right)=\left[\begin{array}{c}
\frac{1}{m V_{a}}(D \sin (\beta) \cos (\mu) \tan (\gamma)+Y \tan (\gamma) \cos (\mu) \cos (\beta)+L(\tan (\beta)+\tan (\gamma) \sin (\mu))+\cdots \\
\cdots+T(\sin (\alpha) \tan (\gamma) \sin (\mu)+\sin (\alpha) \tan (\beta)-\cos (\alpha) \tan (\gamma) \cos (\mu) \sin (\beta))-\frac{g \cos (\gamma) \cos (\mu) \tan (\beta)}{V_{a}} \\
-\frac{1}{m V_{a} \cos (\beta)}(L+T \sin (\alpha))+\frac{g \cos (\gamma) \cos (\mu)}{V_{a} \cos (\beta)} \\
\frac{1}{m V_{a}}(D \sin (\beta)+Y \cos (\beta)-T \sin (\beta) \cos (\alpha))+\frac{g \cos (\gamma) \sin (\mu)}{V_{a}}
\end{array}\right] \\
& \mathbf{G}_{2}\left(\mathbf{x}_{2}\right)=\left[\begin{array}{ccc}
\frac{\cos (\alpha)}{\cos (\beta)} & 0 & \frac{\sin (\alpha)}{\cos (\beta)} \\
-\tan (\beta) \cos (\alpha) & 1 & -\tan (\beta) \sin (\alpha) \\
\sin (\alpha) & 0 & -\cos (\alpha)
\end{array}\right]
\end{aligned}
$$




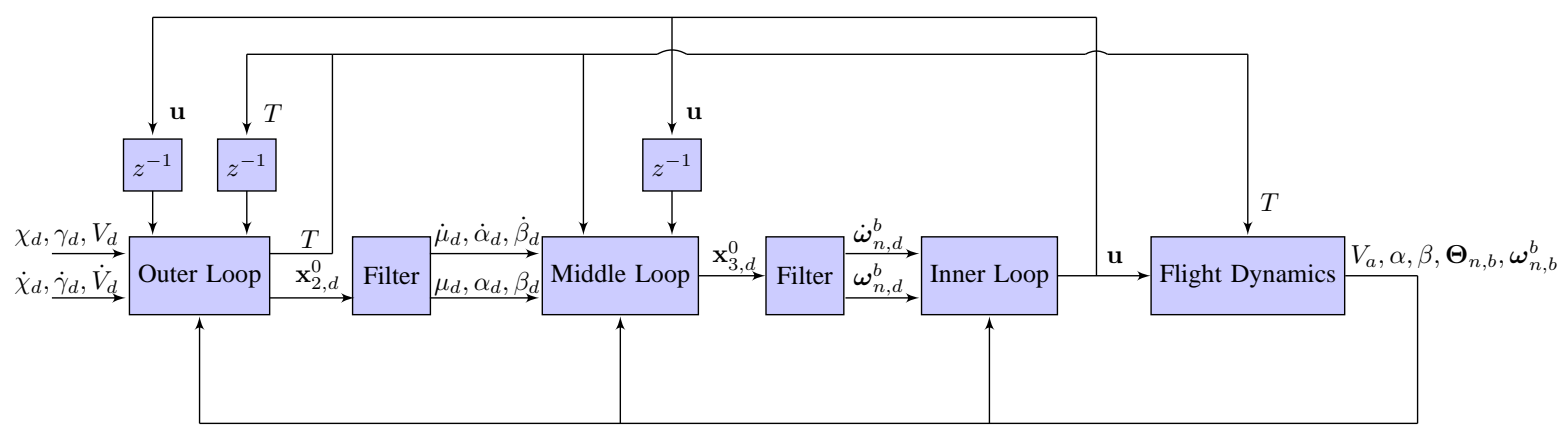

Fig. 2. Command-filtered backstepping control structure.

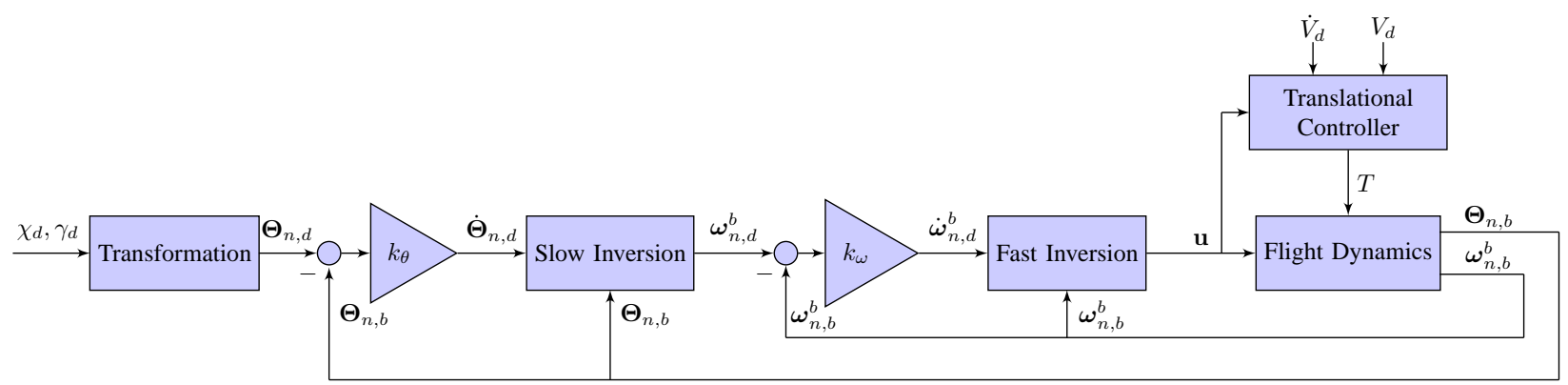

Fig. 3. An inner-outer loop nonlinear dynamic inversion controller showing the main signal paths. Using the angles of attack, the transformation block transforms the desired flight path and course angle to desired Euler angles, which then are tracked using this controller.

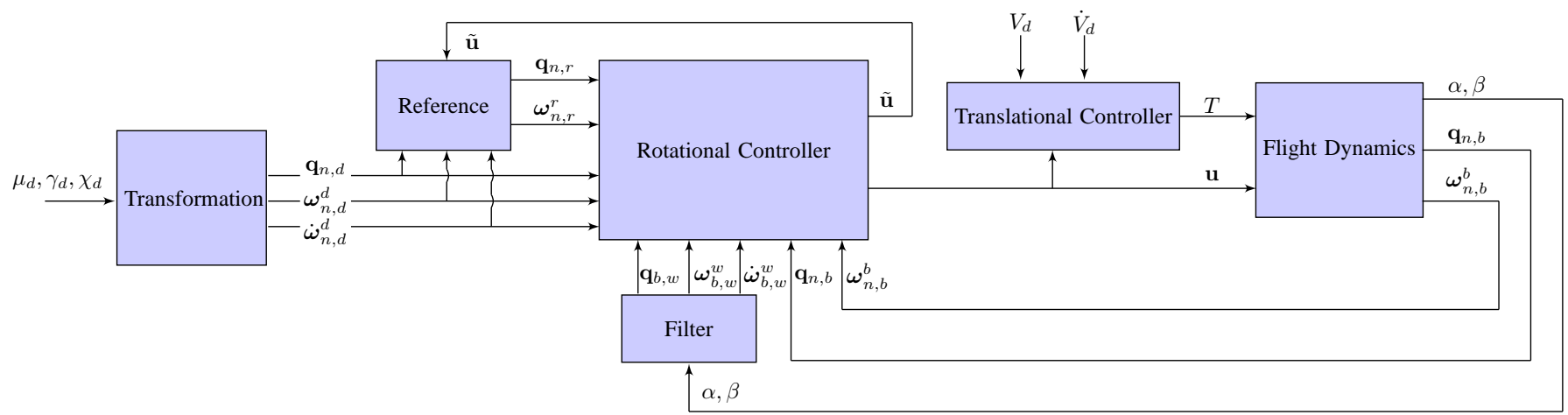

Fig. 4. Decoupled control structure with reference generator showing the main signal paths. In the case when not using the reference, the desired states are used directly by the rotational controller and the reference block can be ignored. The transformation block comprise a linear filter to find the second order derivatives of the desired trajectories, which then allows the calculations of the desired quaternion, angular velocity and acceleration.

of attack $(\alpha)$, the objective can be achieved. Let the lift be decomposed as $L=L_{0}+L_{\alpha} \alpha$, where

$$
\begin{aligned}
L_{0} & =\frac{1}{2} \rho S V_{a}^{2}\left(C_{L_{0}}+\frac{\bar{c}}{2 V_{a}} C_{L_{q}} q+C_{L_{\delta_{e}}} \delta_{e}\right) \\
L_{\alpha} & =\frac{1}{2} \rho S V_{a}^{2} C_{L_{\alpha}}
\end{aligned}
$$

then (24) can be rewritten as

$$
\mathbf{g}_{1}(\cdot)=\left[\begin{array}{c}
\frac{1}{m V_{a} \cos (\gamma)}\left(L_{0}+L_{\alpha} \alpha+T \sin (\alpha)\right) \sin (\mu) \\
\frac{1}{m V_{a}}\left(L_{0}+L_{\alpha} \alpha+T \sin (\alpha)\right) \cos (\mu) \\
\frac{1}{m} T \cos (\beta) \cos (\alpha)
\end{array}\right] .
$$

From the control law, the left-hand side is known, such that its results must be achieved using the available control signals. The two first components of the vector can be seen as Cartesian coordinates that can be transformed into polar coordinates where $L_{0}+L_{\alpha} \alpha+T \sin (\alpha)$ can be seen as the radius. Let a vector of scaled points be defined as

$$
\left[\begin{array}{l}
x_{0} \\
y_{0} \\
z_{0}
\end{array}\right]=\left[\begin{array}{ccc}
m V_{a} \cos (\gamma) & 0 & 0 \\
0 & m V_{a} & 0 \\
0 & 0 & 1
\end{array}\right] \mathbf{g}_{1}(\cdot),
$$

where $\mathbf{g}_{1}(\cdot)$ is from (27), then the desired angle of attack can be found using $\sqrt{x_{0}^{2}+y_{0}^{2}}=L_{0}+L_{\alpha} \alpha_{d}^{0}+T \sin (\alpha)$ as

$$
\alpha_{d}^{0}=\frac{1}{L_{\alpha}}\left(\sqrt{x_{0}^{2}+y_{0}^{2}}-L_{0}-T \sin (\alpha)\right)
$$

while the bank angle can be found as $\mu_{d}^{0}=\operatorname{atan} 2\left(x_{0}, y_{0}\right)$, where $\operatorname{atan} 2(\cdot, \cdot)$ is the four quadrant version of $\tan ^{-1}(\cdot)$. Note that the bank angle will not necessarily converge to zero as $x_{0}$ is a function of $\mathbf{f}_{1}(\cdot)$, which again is a function of the aerodynamic sideforce $Y$. The aerodynamic sideforce contains 
the coefficient $C_{Y_{0}}$, which can be non-zero depending on the shape of the aircraft, as well as sideforces created due to the ailerons and rudder. With the desired bank angle and angle of attack, the thrust can be found directly using (24) and (31) as

$$
T_{d}=\frac{m}{\cos \left(\alpha_{d}^{0}\right) \cos \left(\beta_{d}^{0}\right)} z_{0}
$$

where $\beta_{d}^{0}=0$. The next step now is to design the middle loop, and to ensure that the desired states and their derivatives remain bounded, a command filter will be employed. A command filter with magnitude and rate saturation can be defined as [9]

$$
\begin{aligned}
& \dot{q}_{1}(t)=q_{2}(t) \\
& \dot{q}_{2}(t)=2 \zeta \omega_{n}\left(\sigma_{R}\left(\frac{\omega_{n}^{2}}{2 \zeta \omega_{n}} \sigma_{M}\left(x_{d}^{0}\right)-q_{1}\right)-q_{2}\right)
\end{aligned}
$$

where $\sigma_{R}(\cdot)$ and $\sigma_{M}(\cdot)$ are rate and magnitude saturation functions, $\omega_{n}$ is the natural frequency, $\zeta$ is the relative damping ratio, and $x_{d}^{0}$ is a desired signal (e.g. $\alpha_{d}^{0}$ ). The commandfiltered signals can now be found as $x_{d}=q_{1}$ and $\dot{x}_{d}=q_{2}$ providing the signals for the next step in the backstepping process.

With the desired state vector $\mathbf{x}_{2, d}$ and $\dot{\mathbf{x}}_{2, d}$ generated using a command-filter, the middle loop can be now designed. From (21), a control law can be chosen as

$$
\mathbf{x}_{3, d}^{0}=\mathbf{G}_{2}^{-1}\left(\mathbf{x}_{2}\right)\left(\dot{\mathbf{x}}_{2, d}-\mathbf{f}_{2}(\cdot)-\mathbf{K}_{2}\left(\mathbf{x}_{2}-\mathbf{x}_{2, d}\right)\right)
$$

with $\mathbf{K}_{2}$ as a positive definite gain matrix. Note that even though this is backstepping, the cross-term that would arise through the backstepping process is not included in [10]. By using another command-filter, the desired angular velocity and acceleration $\dot{\mathbf{x}}_{3, d}$ and $\mathbf{x}_{3, d}$ are obtained, allowing a control law to be designed as

$$
\mathbf{u}=\mathbf{G}^{-1}(\mathbf{x})\left(\mathbf{J} \dot{\mathbf{x}}_{3, d}-\mathbf{f}_{3}(\cdot)-\mathbf{K}_{3}\left(\mathbf{x}_{3}-\mathbf{x}_{3, d}\right)\right)
$$

where $\mathbf{K}_{3}$ is another positive definite gain matrix. Using original notation, the control law can be written as

$$
\begin{aligned}
\mathbf{u}= & \mathbf{G}^{-1}(\mathbf{x})\left(\mathbf{J} \dot{\boldsymbol{\omega}}_{n, d}^{b}+\mathbf{S}\left(\boldsymbol{\omega}_{n, b}^{b}\right) \mathbf{J} \boldsymbol{\omega}_{n, b}^{b}-\mathbf{f}(\mathbf{x})\right. \\
& \left.+\mathbf{D}(\mathbf{x}) \boldsymbol{\omega}_{n, b}^{b}-\mathbf{K}_{3}\left(\boldsymbol{\omega}_{n, b}^{b}-\boldsymbol{\omega}_{n, d}^{b}\right)\right)
\end{aligned}
$$

where it is evident that the backstepping and command filter provides the desired angular acceleration and angular velocity.

\section{B. Nonlinear Dynamic Inversion}

Based on [20], an inner-outer loop controller can be designed using Euler angles. Given the input $\mu_{d}, \gamma_{d}$ and $\chi_{d}$, representing the bank, flight path and heading angles, the desired Euler angles can be found as $\boldsymbol{\Theta}_{n, d}=\left[\begin{array}{lll}\phi_{d} & \theta_{d} & \psi_{d}\end{array}\right]^{\top}$ where $\phi_{d}=\mu_{d}, \theta_{d}=\gamma_{d}+\alpha$ and $\psi_{d}=\chi_{d}-\beta$. A similar definition has been applied in [21] to design desired angles that can be tracked using a rotational controller. The underlying assumptions are that the longitudinal and lateral motion are decoupled and can be treated independently, something that works satisfactory for most cases. Nonlinear dynamic inversion requires a desired angular acceleration to invert the system (12) with regards to the deflection angles, resulting in a control signal that produces a given desired angular acceleration.Tracking of desired angles can then be achieved by properly defining the desired angular acceleration. First the outer loop can be designed, which is considered slowly varying. Based on the errors between the actual Euler angles and their desired ones, the desired angular rates are found as

$$
\dot{\boldsymbol{\Theta}}_{n, d}=k_{\theta}\left(\boldsymbol{\Theta}_{n, d}-\boldsymbol{\Theta}_{n, b}\right)
$$

where $k_{\theta}$ is a positive gain. Note that this is a simple proportional controller, and other implementations using PI or PID controllers may also be used for this. Based on the desired angular rates, the desired angular velocity can be found as

$$
\boldsymbol{\omega}_{n, d}^{b}=\mathbf{T}^{-1}\left(\boldsymbol{\Theta}_{n, b}\right) \dot{\boldsymbol{\Theta}}_{n, d} .
$$

Note that $\mathbf{T}\left(\boldsymbol{\Theta}_{n, b}\right)$ is singular when $\theta= \pm \frac{\pi}{2}$. The desired angular acceleration can now be obtained as

$$
\dot{\boldsymbol{\omega}}_{n, d}^{b}=k_{\omega}\left(\boldsymbol{\omega}_{n, d}^{b}-\boldsymbol{\omega}_{n, b}^{b}\right)
$$

where $k_{\omega}$ is another positive gain. By using nonlinear dynamic inversion, the deflection angles are now found through inversion of (12) as

$$
\begin{aligned}
\mathbf{u}= & \mathbf{G}^{-1}(\mathbf{x})\left(\mathbf{J} \dot{\boldsymbol{\omega}}_{n, d}^{b}+\mathbf{S}\left(\boldsymbol{\omega}_{n, b}^{b}\right) \mathbf{J} \boldsymbol{\omega}_{n, b}^{b}-\mathbf{f}(\mathbf{x})\right. \\
& \left.+\mathbf{D}(\mathbf{x}) \boldsymbol{\omega}_{n, b}^{b}\right),
\end{aligned}
$$

providing a control law that enables an aircraft to track desired angles.

\section{Decoupled Approach}

The control problem of pointing the wind frame in a desired direction and move with a positive desired airspeed can also be achieved using a quaternion representation. Let the desired trajectory be defined through $\mathbf{q}_{n, d}, \boldsymbol{\omega}_{n, d}^{d}, \dot{\boldsymbol{\omega}}_{n, d}^{d} \in \mathcal{L}_{\infty}$, then the quaternion error can be defined as $\mathbf{q}_{d, w}=\mathbf{q}_{d, n} \otimes$ $\mathbf{q}_{n, b} \otimes \mathbf{q}_{b, w}$ where $\mathbf{q}_{b, w}$ represents the rotation from the wind frame to the body frame and can be defined using the angle of attack and sideslip as $\mathbf{q}_{b, w}=\mathbf{q}_{b, s} \otimes \mathbf{q}_{s, w}$ where $\mathbf{q}_{b, s}=\left[\begin{array}{llll}\cos \left(\frac{\alpha}{2}\right) & 0 & -\sin \left(\frac{\alpha}{2}\right) & 0\end{array}\right]^{\top}$ and $\mathbf{q}_{s, w}=$ $\left[\begin{array}{llll}\cos \left(\frac{\beta}{2}\right) & 0 & 0 & \sin \left(\frac{\beta}{2}\right)\end{array}\right]^{\top}$. Furthermore, the angular velocity and acceleration of the wind frame relative to the body frame are found as

$$
\begin{aligned}
& \boldsymbol{\omega}_{b, w}^{w}=\left[\begin{array}{c}
-\dot{\alpha} \sin (\beta) \\
-\dot{\alpha} \cos (\beta) \\
\dot{\beta}
\end{array}\right] \\
& \dot{\boldsymbol{\omega}}_{b, w}^{w}=\left[\begin{array}{c}
-\ddot{\alpha} \sin (\beta)-\dot{\alpha} \dot{\beta} \cos (\beta) \\
-\ddot{\alpha} \cos (\beta)+\dot{\alpha} \dot{\beta} \sin (\beta) \\
\ddot{\beta}
\end{array}\right] .
\end{aligned}
$$

From (44) it is observed that the angular acceleration of the wind frame relative to the body frame comprises the second order derivatives of the angle of attack and sideslip angle. These angles are not available for measurements, but the angle of attack and sideslip can be measured using a 5-hole probe. This allows estimates of their second derivatives to be found using, e.g. a linear filter, a Kalman filter [11], or using sliding mode differentiator [22]. While each of the three methods work very well for finding the higher order derivatives, the 
authors have obtained the best results using a simple linear filter. A linear filter can be proposed as (cf. [23])

$$
\begin{aligned}
\dot{\hat{\mathbf{x}}}_{r} & =\mathbf{A} \hat{\mathbf{x}}_{r}+\mathbf{B} r \\
\mathbf{A} & =\left[\begin{array}{ccc}
-(2 \eta+1) \Omega & -(2 \eta+1) \Omega^{2} & -\Omega^{3} \\
0 & 1 & 0 \\
0 & 0 & 1
\end{array}\right] \\
\mathbf{B} & =\left[\begin{array}{lll}
\Omega^{3} & 0 & 0
\end{array}\right]^{\top}
\end{aligned}
$$

where $\hat{\mathbf{x}}_{r}=\left[\begin{array}{lll}\ddot{\hat{r}} & \dot{\hat{r}} & \hat{r}\end{array}\right]^{\top}$ with $r=\alpha$ in the case of the angle of attack, and similarly for the sideslip angle. The relative damping ratio, $\eta$, and natural frequency, $\Omega$, can be used to tune the performance of the filter, but in this work it is simply set as $\eta=\Omega=1$.

This filter allows the rotational and translational dynamics to become decoupled as the rotational controller can be easily design from the error dynamics where all the states now are available. The output from the rotational controller can then serve as input to the translational controller when calculating the desired thrust, something that again can be designed using standard Lyapunov methods.

1) Desired Quaternion, Angular Velocity and Acceleration: It is typical to define a desired bank, flight path and course angle as well as a desired airspeed. In order to find the desired quaternion, angular velocity and angular acceleration, the desired angles can be sent through a linear filter as shown above, enabling the estimation of $\boldsymbol{\Theta}_{n, d}=\left[\begin{array}{lll}\mu_{d} & \gamma_{d} & \chi_{d}\end{array}\right]^{\top}$ and its first and second order derivatives. Now to find the desired quaternion, $\mathbf{q}_{n, d}$, it can be constructed using the desired angles as described in [23, p. 32]. To find the desired angular velocity and acceleration, they can be found as $\boldsymbol{\omega}_{n, d}^{d}=\mathbf{T}^{-1}\left(\boldsymbol{\Theta}_{n, d}\right) \dot{\boldsymbol{\Theta}}_{n, d}$ and $\dot{\boldsymbol{\omega}}_{n, d}^{d}=\dot{\mathbf{T}}^{-1}\left(\boldsymbol{\Theta}_{n, d}\right) \dot{\boldsymbol{\Theta}}_{n, d}+$ $\mathbf{T}^{-1}\left(\boldsymbol{\Theta}_{n, d}\right) \ddot{\boldsymbol{\Theta}}_{n, d}$. There are naturally many other ways of finding the desired states, but this is the approach used in this paper.

2) Backstepping Control using Decoupled Method: A backstepping controller can now be designed to make the attitude and angular velocity errors go to zero. Some preliminary results on this approach is given in [24], which shows how to decouple the translational and rotational dynamics, and how to control the aircraft using a sliding surface controller. This section presents a nonlinear backstepping controller using the decoupled dynamics, where the control objective is to make $\mathbf{q}_{d, w} \rightarrow\left[\begin{array}{llll} \pm 1 & 0 & 0 & 0\end{array}\right]^{\top}$ and $\boldsymbol{\omega}_{d, w}^{b} \rightarrow \mathbf{0}$. First let an error function be defined as (cf. [25])

$$
\mathbf{e}_{q \pm}=\left[\begin{array}{c}
1 \mp \eta_{d, w} \\
\boldsymbol{\epsilon}_{d, w}
\end{array}\right]
$$

with the kinematics as

$$
\begin{aligned}
\dot{\mathbf{e}}_{q \pm} & =\mathbf{T}_{e}\left(\mathbf{e}_{q \pm}\right) \mathbf{R}_{b}^{w} \boldsymbol{\omega}_{d, w}^{b} \\
\mathbf{T}_{e}\left(\mathbf{e}_{q \pm}\right) & =\frac{1}{2}\left[\begin{array}{c} 
\pm \boldsymbol{\epsilon}_{d, w}^{\top} \\
\eta_{d, w} \mathbf{I}+\mathbf{S}\left(\boldsymbol{\epsilon}_{d, w}\right)
\end{array}\right]
\end{aligned}
$$

and where $\boldsymbol{\omega}_{d, w}^{b}=\boldsymbol{\omega}_{n, b}^{b}-\mathbf{R}_{d}^{b} \boldsymbol{\omega}_{n, d}^{d}+\mathbf{R}_{w}^{b} \boldsymbol{\omega}_{b, w}^{w}$. By introducing this error function, the equilibrium point becomes shifted to zero such that the objective is to make $\left(\mathbf{e}_{q \pm}, \boldsymbol{\omega}_{d, w}^{b}\right) \rightarrow(\mathbf{0}, \mathbf{0})$.
The control problem can be made easier through the following assumption.

Assumption 1: Assume that $\left.\operatorname{sgn}\left(\eta_{d, w}\left(t_{0}\right)\right)=\operatorname{sgn}\left(\eta_{d, w}\right)(t)\right)$. This assumption divides the rotational sphere into two halves, allowing the control design to focus only on one of the two equilibrium points (physically the same orientation, but mathematically different). This simplifies the controller design and will work very well for most cases. What might happen, is that if the aircraft starts with an initial condition that is far away from the correct equilibrium point, it might perform a $360^{\circ}$ maneuver to reach the selected equilibrium point, but in practice this is not an issue. The reader is referred to the work by [26] on the topic of the unwinding phenomenon for more details regarding this. By using Assumption 1 the following inequality holds ( $c f$. [25] and [27])

$$
\mathbf{e}_{q \pm}^{\top} \mathbf{T}_{e} \mathbf{T}_{e}^{\top} \mathbf{e}_{q \pm} \geq \frac{1}{8} \mathbf{e}_{q \pm}^{\top} \mathbf{e}_{q \pm}
$$

Also note that $\mathbf{T}_{e}^{\top} \mathbf{e}_{q}=\frac{1}{2} \boldsymbol{\epsilon}_{d, w}$. Now consider the positive equilibrium point such that $\mathbf{e}_{q}:=\mathbf{e}_{q+}$ and let a Lyapunov function candidate be chosen as $V_{1}:=\frac{1}{2} \mathbf{e}_{q}^{\top} \mathbf{e}_{q}$, which can be differentiated using (49) as

$$
\dot{V}_{1}=\mathbf{e}_{q}^{\top} \mathbf{T}_{e} \mathbf{R}_{b}^{w} \boldsymbol{\omega}_{d, w}^{b} .
$$

The angular velocity error can now be treated as a virtual control signal and chosen as

$$
\boldsymbol{\omega}_{d, w}^{b}=-k_{q} \mathbf{R}_{w}^{b} \mathbf{T}_{e}^{\top} \mathbf{e}_{q}+\mathbf{z}
$$

where $k_{q}>0$ is a positive gain and $\mathbf{z}$ is a new variable that arise through the backstepping process. Inserting (53) into (52) and using results in

$$
\dot{V}_{1} \leq-\frac{k_{q}}{8} \mathbf{e}_{q}^{\top} \mathbf{e}_{q}+\mathbf{e}_{q}^{\top} \mathbf{T}_{e} \mathbf{R}_{b}^{w} \mathbf{z}
$$

which is negative definite as long as $\mathbf{z}=\mathbf{0}$. The dynamics of the new variable is found from (53) by using (12) as

$$
\begin{aligned}
\mathbf{J} \dot{\mathbf{z}}= & -\mathbf{S}\left(\boldsymbol{\omega}_{n, b}^{b}\right) \mathbf{J} \boldsymbol{\omega}_{n, b}^{b}+\mathbf{f}(\mathbf{x})-\mathbf{D}(\mathbf{x}) \boldsymbol{\omega}_{n, b}^{b}+\mathbf{G}(\mathbf{x}) \mathbf{u} \\
& +\mathbf{J S}\left(\boldsymbol{\omega}_{n, b}^{b}\right) \mathbf{R}_{d}^{b} \boldsymbol{\omega}_{n, d}^{d}-\mathbf{J} \mathbf{R}_{d}^{b} \dot{\boldsymbol{\omega}}_{n, d}^{d}+\mathbf{J} \mathbf{R}_{w}^{b} \dot{\boldsymbol{\omega}}_{b, w}^{w} \\
& +\frac{k_{q}}{2} \mathbf{J R}_{w}^{b} \mathbf{S}\left(\boldsymbol{\omega}_{b, w}^{w}\right) \boldsymbol{\epsilon}_{d, w}+\frac{k_{q}}{2} \mathbf{J R}_{w}^{b} \dot{\boldsymbol{\epsilon}}_{d, w}
\end{aligned}
$$

A second Lyapunov function candidate can now be chosen as $V_{2}:=V_{1}+\frac{1}{2} \mathbf{z}^{\top} \mathbf{J z}$ and differentiated using (52) and (55) as

$$
\begin{aligned}
\dot{V}_{2} \leq & -\frac{k_{q}}{8} \mathbf{e}_{q}^{\top} \mathbf{e}_{q}+\mathbf{e}_{q}^{\top} \mathbf{T}_{e} \mathbf{R}_{b}^{w} \mathbf{z} \\
& +\mathbf{z}^{\top}\left(-\mathbf{S}\left(\boldsymbol{\omega}_{n, b}^{b}\right) \mathbf{J} \boldsymbol{\omega}_{n, b}^{b}+\mathbf{f}(\mathbf{x})-\mathbf{D}(\mathbf{x}) \boldsymbol{\omega}_{n, b}^{b}+\mathbf{G}(\mathbf{x}) \mathbf{u}\right. \\
& +\mathbf{J S}\left(\boldsymbol{\omega}_{n, b}^{b}\right) \mathbf{R}_{d}^{b} \boldsymbol{\omega}_{n, d}^{d}-\mathbf{J} \mathbf{R}_{d}^{b} \dot{\boldsymbol{\omega}}_{n, d}^{d}+\mathbf{J} \mathbf{R}_{w}^{b} \dot{\boldsymbol{\omega}}_{b, w}^{w} \\
& \left.+\frac{k_{q}}{2} \mathbf{J} \mathbf{R}_{w}^{b} \mathbf{S}\left(\boldsymbol{\omega}_{b, w}^{w}\right) \boldsymbol{\epsilon}_{d, w}+\frac{k_{q}}{2} \mathbf{J} \mathbf{R}_{w}^{b} \dot{\boldsymbol{\epsilon}}_{d, w}\right)
\end{aligned}
$$

The control law can now be chosen as

$$
\begin{aligned}
\mathbf{u}= & \mathbf{G}^{-1}(\mathbf{x})\left(\mathbf{J} \mathbf{R}_{d}^{b} \dot{\boldsymbol{\omega}}_{n, d}^{d}-\mathbf{J S}\left(\boldsymbol{\omega}_{n, b}^{b}\right) \mathbf{R}_{d}^{b} \boldsymbol{\omega}_{n, d}^{d}-\mathbf{J} \mathbf{R}_{w}^{b} \dot{\boldsymbol{\omega}}_{b, w}^{w}\right. \\
& +\mathbf{S}\left(\boldsymbol{\omega}_{n, b}^{b}\right) \mathbf{J} \boldsymbol{\omega}_{n, b}^{b}-\mathbf{f}(\mathbf{x})+\mathbf{D}(\mathbf{x}) \boldsymbol{\omega}_{n, b}^{b}-\frac{1}{2} \mathbf{R}_{w}^{b} \boldsymbol{\epsilon}_{d, w} \\
& \left.-\frac{k_{q}}{2} \mathbf{J} \mathbf{R}_{w}^{b} \mathbf{S}\left(\boldsymbol{\omega}_{b, w}^{w}\right) \boldsymbol{\epsilon}_{d, w}-\frac{k_{q}}{2} \mathbf{J} \mathbf{R}_{w}^{b} \dot{\boldsymbol{\epsilon}}_{d, w}-\mathbf{K}_{z} \mathbf{z}\right)
\end{aligned}
$$


where $\mathbf{K}_{z}=\mathbf{K}_{z}^{\top}>\mathbf{0}$ is a gain matrix, and by inserting (57) into (56), it results in $\dot{V}_{2} \leq-\frac{k_{q}}{8}\left\|\mathbf{e}_{q}\right\|^{2}-k_{z}\|\mathbf{z}\|^{2}$, which is negative definite and where $k_{z}$ is the smallest eigenvalue of $\mathbf{K}_{z}$. By applying Theorem 4.10 from [28], it follows that the origin $\left(\mathbf{e}_{q}, \mathbf{z}\right)=(\mathbf{0}, \mathbf{0})$ is uniformly exponentially stable. As $\left(\mathbf{e}_{q}, \mathbf{z}\right) \rightarrow(\mathbf{0}, \mathbf{0})$, it follows from (53) that $\boldsymbol{\omega}_{d, w}^{b} \rightarrow \mathbf{0}$ thereby completing the control objective. A similar proof can be done for the negative equilibrium point.

3) Decoupled Approach with Reference: It is well established that ailerons are much better than the rudder for controlling the heading. By using nonlinear dynamic inversion or the decoupled method, the heading error becomes mapped to the rudder, while the command-filtered backstepping approach uses the bank angle as a state variable that naturally becomes mapped to the ailerons. To achieve comparable results with the command-filtered backstepping (or better), a reference signal will be designed in this section. The results are inspired by pseudo-control hedging by [12], such that the reference deviates whenever the actuators are in saturation to avoid the deterioration of the adaptive update laws. While [12] (and later [29]) designed the reference from the angular velocity level, an adaptive approach to this is presented in [30], which designs the reference from the quaternion level. One very interesting result of starting at the quaternion level that has not been studied before, is that it allows a mapping of control deficiency from one actuator to another, thereby increasing the control authority of the aircraft.

First a reference signal can be defined as

$$
\dot{\mathbf{q}}_{n, r}=\frac{1}{2} \mathbf{T}\left(\mathbf{q}_{n, r}\right)\left[\begin{array}{c}
0 \\
\boldsymbol{\omega}_{n, r}^{r}
\end{array}\right]
$$

where the angular acceleration $\dot{\boldsymbol{\omega}}_{n, r}^{r}$ is to be designed. The objective of the reference signal is to track the desired trajectory, but deviate whenever the actuators are in saturation. Let the attitude error be defined as $\mathbf{q}_{r, d}=\mathbf{q}_{r, n} \otimes \mathbf{q}_{n, d}$ and an error function as $\mathbf{e}_{q_{r}}=\left[\begin{array}{ll}1-\eta_{d, r} & \boldsymbol{\epsilon}_{d, r}^{\top}\end{array}\right]^{\top}$ where only the positive equilibrium point is considered. The error between the reference trajectory and the desired frame has the kinematics

$$
\dot{\mathbf{e}}_{q_{r}}=\mathbf{T}_{e_{r}}\left(\mathbf{e}_{q_{r}}\right)\left(\boldsymbol{\omega}_{n, r}^{r}-\mathbf{R}_{d}^{r} \boldsymbol{\omega}_{n, d}^{d}\right),
$$

which can be driven to zero using backstepping similarly as before. Let $\boldsymbol{\omega}_{n, r}^{r}=\mathbf{R}_{d}^{r} \boldsymbol{\omega}_{n, d}^{d}-k_{1} \mathbf{T}_{e_{r}}^{\top} \mathbf{e}_{q_{r}}+\mathbf{z}_{r}$, giving the dynamics of the new variable as

$$
\dot{\mathbf{z}}_{r}=\dot{\boldsymbol{\omega}}_{n, r}^{r}+\mathbf{S}\left(\boldsymbol{\omega}_{n, r}^{r}\right) \mathbf{R}_{d}^{r} \boldsymbol{\omega}_{n, d}^{d}-\mathbf{R}_{d}^{r} \dot{\boldsymbol{\omega}}_{n, d}^{d}+\frac{k_{1}}{2} \dot{\boldsymbol{\epsilon}}_{d, r} .
$$

The angular acceleration of the reference trajectory relative to NED can now be chosen as

$$
\begin{aligned}
\dot{\boldsymbol{\omega}}_{n, r}^{r}= & \mathbf{R}_{d}^{r} \dot{\boldsymbol{\omega}}_{n, d}^{d}-\mathbf{S}\left(\boldsymbol{\omega}_{n, r}^{r}\right) \mathbf{R}_{d}^{r} \boldsymbol{\omega}_{n, d}^{d} \\
& -\frac{k_{1}}{2} \dot{\boldsymbol{\epsilon}}_{d, r}-\frac{1}{2} \boldsymbol{\epsilon}_{d, r}-k_{2} \mathbf{z}_{r}+\boldsymbol{\xi}:=\mathbf{\Upsilon}+\boldsymbol{\xi}
\end{aligned}
$$

where $\boldsymbol{\xi}$ is a bounded function to be designed. Given a Lyapunov function candidate as $V_{3}:=\frac{1}{2} \mathbf{e}_{q_{r}}^{\top} \mathbf{e}_{q_{r}}+\frac{1}{2} \mathbf{z}_{r}^{\top} \mathbf{z}_{r}$ it can be differentiated and by inserting (61) results in

$$
\dot{V}_{3} \leq-\frac{k_{1}}{8}\left\|\mathbf{e}_{q_{r}}\right\|^{2}-k_{2}\left\|\mathbf{z}_{r}\right\|^{2}+\mathbf{z}_{r}^{\top} \boldsymbol{\xi},
$$

indicating that the origin $\left(\mathbf{e}_{q_{r}}, \mathbf{z}_{r}\right)=(\mathbf{0}, \mathbf{0})$ is uniformly asymptotically stable as long as $\boldsymbol{\xi}=\mathbf{0}$. By allowing the function $\boldsymbol{\xi}$ to be a bounded converging function, i.e. $\boldsymbol{\xi} \rightarrow \mathbf{0}$ as $t \rightarrow \infty$, then it follows by applying Definition 4.7 in [28] that the system (59)-(60) is input-to-state stable, where $\left(\mathbf{e}_{q_{r}}, \mathbf{z}_{r}\right) \rightarrow(\mathbf{0}, \mathbf{0})$ as $\boldsymbol{\xi} \rightarrow \mathbf{0}$. Now to account for actuator saturation, let the actuator signal be rewritten as $\mathbf{u}=\sigma(\mathbf{u})+\tilde{\mathbf{u}}$, where $\sigma(\mathbf{u})$ denotes the saturated signal (the available signal), while $\tilde{\mathbf{u}}$ is the difference between the commanded and saturated signal (i.e. command deficiency). The quaternion error can now be redefined as $\mathbf{q}_{r, w}=\mathbf{q}_{r, n} \otimes \mathbf{q}_{n, b} \otimes \mathbf{q}_{b, w}$ with an error function as $\mathbf{e}_{q 2}=\left[\begin{array}{ll}1-\eta_{r, w} & \boldsymbol{\epsilon}_{r, w}^{\top}\end{array}\right]^{\top}$ (only considering the positive equilibrium point). The error kinematics become

$$
\dot{\mathbf{e}}_{q 2}=\mathbf{T}_{e 2}\left(\mathbf{e}_{q 2}\right) \mathbf{R}_{b}^{w} \boldsymbol{\omega}_{r, w}^{b}
$$

where $\boldsymbol{\omega}_{r, w}^{b}=\boldsymbol{\omega}_{n, b}^{b}-\mathbf{R}_{r}^{b} \boldsymbol{\omega}_{n, r}^{r}+\mathbf{R}_{w}^{b} \boldsymbol{\omega}_{b, w}^{w}$. The control objective is therefore to make $\left(\mathbf{e}_{q 2}, \boldsymbol{\omega}_{r, w}^{b}\right) \rightarrow(\mathbf{0}, \mathbf{0})$ while accounting for the actuator limitations. Through backstepping, the angular velocity can again be used as a virtual control signal and chosen as $\boldsymbol{\omega}_{r, w}^{b}=-k_{3} \mathbf{R}_{w}^{b} \mathbf{T}_{e 2}^{\top} \mathbf{e}_{q 2}+\mathbf{z}_{2}$ where $k_{3}>0$ is a gain and $\mathbf{z}_{2}$ is a new variable with the dynamics using (12) and (61) as

$$
\begin{aligned}
\mathbf{J} \dot{\mathbf{z}}_{2}= & -\mathbf{S}\left(\boldsymbol{\omega}_{n, b}^{b}\right) \mathbf{J} \boldsymbol{\omega}_{n, b}^{b}+\mathbf{f}(\mathbf{x})-\mathbf{D}(\mathbf{x}) \boldsymbol{\omega}_{n, b}^{b}+\mathbf{G}(\mathbf{x}) \sigma(\mathbf{u}) \\
& +\mathbf{G}(\mathbf{x}) \tilde{\mathbf{u}}+\mathbf{J S}\left(\boldsymbol{\omega}_{n, b}^{b}\right) \mathbf{R}_{r}^{b} \boldsymbol{\omega}_{n, r}^{r}-\mathbf{J} \mathbf{R}_{r}^{b}(\boldsymbol{\Upsilon}+\boldsymbol{\xi})+\mathbf{J R}_{w}^{b} \dot{\boldsymbol{\omega}}_{b, w}^{w} \\
& +\frac{k_{3}}{2} \mathbf{J} \mathbf{R}_{w}^{b} \mathbf{S}\left(\boldsymbol{\omega}_{b, w}^{w}\right) \boldsymbol{\epsilon}_{r, w}+\frac{k_{3}}{2} \mathbf{J R}_{w}^{b} \dot{\boldsymbol{\epsilon}}_{r, w} .
\end{aligned}
$$

The control deficiency can now be removed from the error dynamics by choosing

$$
\boldsymbol{\xi}:=\mathbf{R}_{b}^{r} \mathbf{J}^{-1} \mathbf{G}(\mathbf{x}) \tilde{\mathbf{u}},
$$

which will be part of the reference signal (61). This means that the reference signal will deviate from the desired trajectory whenever the actuators are in saturation. By assuming that the desired trajectory is a feasible trajectory for the aircraft, it is reasonable to assume that the control deficiency, $\tilde{\mathbf{u}}$, is bounded, and will go to zero in finite time making $\boldsymbol{\xi} \rightarrow \mathbf{0}$. Note that this assumption is paramount for the design. Now consider the case where the rudder is in saturation such that $\left|\tilde{\delta}_{r}\right|>0$, then the function can be expanded as

$$
\boldsymbol{\xi}=\frac{1}{2} \rho S V_{a}^{2} \mathbf{R}_{b}^{r} \mathbf{J}^{-1}\left[\begin{array}{ccc}
b C_{l_{\delta_{a}}} & 0 & b C_{l_{\delta_{r}}} \\
0 & \bar{c} C_{m_{\delta_{e}}} & 0 \\
b C_{n_{\delta_{a}}} & 0 & b C_{n_{\delta_{r}}}
\end{array}\right]\left[\begin{array}{c}
0 \\
0 \\
\tilde{\delta}_{r}
\end{array}\right]
$$

where it is obvious that the control deficiency becomes mapped onto the $x$ axis, creating rolling commands in the reference trajectory such that the aircraft starts to roll. The inverse of the inertia matrix and the rotation matrix also contribute to map the control deficiencies to the other axes.

The control law can now be designed following the same procedure as in Section III-C2 With basis in the Lyapunov function candidate $V_{4}:=\frac{1}{2} \mathbf{e}_{q 2}^{\top} \mathbf{e}_{q 2}+\frac{1}{2} \mathbf{z}_{2}^{\top} \mathbf{J} \mathbf{z}_{2}$, a control law can be chosen as

$$
\begin{aligned}
\sigma(\mathbf{u})= & \mathbf{G}^{-1}(\mathbf{x})\left(\mathbf{J} \mathbf{R}_{r}^{b} \boldsymbol{\Upsilon}-\mathbf{J S}\left(\boldsymbol{\omega}_{n, b}^{b}\right) \mathbf{R}_{r}^{b} \boldsymbol{\omega}_{n, r}^{r}-\mathbf{J} \mathbf{R}_{w}^{b} \dot{\boldsymbol{\omega}}_{b, w}^{w}\right. \\
& +\mathbf{S}\left(\boldsymbol{\omega}_{n, b}^{b}\right) \mathbf{J} \boldsymbol{\omega}_{n, b}^{b}-\mathbf{f}(\mathbf{x})+\mathbf{D}(\mathbf{x}) \boldsymbol{\omega}_{n, b}^{b}-\frac{1}{2} \mathbf{R}_{w}^{b} \boldsymbol{\epsilon}_{r, w} \\
& \left.-\frac{k_{3}}{2} \mathbf{J} \mathbf{R}_{w}^{b} \mathbf{S}\left(\boldsymbol{\omega}_{b, w}^{w}\right) \boldsymbol{\epsilon}_{r, w}-\frac{k_{3}}{2} \mathbf{J} \mathbf{R}_{w}^{b} \dot{\boldsymbol{\epsilon}}_{r, w}-\mathbf{K}_{4} \mathbf{z}_{2}\right)
\end{aligned}
$$


where $\mathbf{K}_{4}=\mathbf{K}_{4}^{\top}>\mathbf{0}$ is a gain matrix. By inserting (67) into the derivative of the Lyapunov function candidate, it results in $\dot{V}_{4} \leq-\frac{k_{3}}{8}\left\|\mathbf{e}_{q 2}\right\|^{2}-k_{4}\left\|\mathbf{z}_{2}\right\|^{2}$ with $k_{4}$ as the smallest eigenvalue of $\mathbf{K}_{4}$, and it follows that origin $\left(\mathbf{e}_{q 2}, \boldsymbol{\omega}_{r, w}^{b}\right)=(\mathbf{0}, \mathbf{0})$ is uniformly exponentially stable. Note that even though the wind frame will converge exponentially fast to the reference trajectory, the reference trajectory will diverge whenever the actuators are in saturation. This moves the saturation problem from the controller to the reference with the gained advantage of increased control authority.

Remark 1: The generated moments from the deflection angles are functions of the airspeed. This means that at low airspeeds, the actuators tend to go into saturation (due to stalling), while at high airspeeds the required moments can be generated using small deflection angles. Any of the control laws presented in this paper require the inverse of the $\mathbf{G}(\mathbf{x})$ matrix, which can be written as

$$
\mathbf{G}^{-1}(\mathbf{x})=\frac{2}{\rho V_{a}^{2}}\left[\begin{array}{ccc}
b C_{l_{\delta_{a}}} & 0 & b C_{l_{\delta_{r}}} \\
0 & \bar{c} C_{m_{\delta_{e}}} & 0 \\
b C_{n_{\delta_{a}}} & 0 & b C_{n_{\delta_{r}}}
\end{array}\right]^{-1} .
$$

From (68) it follows that the right hand side of the control laws can be made arbitrarily small by increasing the airspeed, thereby keeping the deflection angles within their linear region. This idea is used in [31] by designing a reference airspeed that deviates from the desired airspeed whenever the deflection angles are in saturation. This addresses the saturation problem of the deflection angles by using the speed, something that represents another way to further increase the control authority of the aircraft.

4) Translational Controller: The objective of the translational controller is to make the airspeed tracking error go to zero. Let a desired speed profile be defined through $V_{d}, \dot{V}_{d} \in$ $\mathcal{L}_{\infty}$ and assume that $V_{a}, u>0 \forall t \geq t_{0}$. A Lyapunov function candidate can then be chosen as $V_{5}:=\frac{1}{2} \tilde{V}^{2}>0 \quad \forall \tilde{V} \neq 0$ and differentiated using equation (9) as

$$
\dot{V}_{5}=\tilde{V}\left(\frac{u}{m V_{a}} T+\frac{\left(\mathbf{v}_{r}^{b}\right)^{\top}}{V_{a}}\left(\frac{1}{m} \mathbf{R}_{w}^{b} \mathbf{f}_{\text {aero }}^{w}+\mathbf{R}_{n}^{b} \mathbf{f}_{g}^{n}\right)-\dot{V}_{d}\right) .
$$

A speed controller can now be chosen as

$$
T=\frac{m V_{a}}{u}\left(\dot{V}_{d}-k_{p} \tilde{V}-\frac{\left(\mathbf{v}_{r}^{b}\right)^{\top}}{V_{a}}\left(\frac{1}{m} \mathbf{R}_{w}^{b} \mathbf{f}_{a e r o}^{w}+\mathbf{R}_{n}^{b} \mathbf{f}_{g}^{n}\right)\right),
$$

resulting in $\dot{V}_{5}=-k_{p} \tilde{V}^{2}$, which is negative definite. It follows that the origin $\tilde{V}=0$ is uniformly exponentially stable, such that the airspeed will exponentially track the desired airspeed as long as the thrust is not in saturation. This controller is used for both decoupled methods and the NDI to make the speed error go to zero.

\section{COMPARISON}

The following simulations assume that the aircraft is equipped with a 5-hole probe providing the airspeed and angles of attack, an inertial navigation system providing the orientation and angular velocity, while a GPS provides the position of the aircraft (not really needed here; but is natural to expect from a navigation system and is an essential part of a guidance system). Four different scenarios are considered:
- Performing a $180^{\circ}$ maneuver with perfect knowledge of states and models.

- Performing a $180^{\circ}$ maneuver when exposed to sensor noise.

- Tracking an aggressive maneuver when exposed to wind gusts and wind shears.

- Tracking an aggressive maneuver when exposed to wind gusts and wind shears with modeling imperfections.

The objective is to gauge how each of the different control laws behave to the different scenarios. The simulations consider a fixed-wing UAV with the parameters as presented in Appendix A, with the deflection angles bounded as $-0.3491 \leq \delta_{a}, \delta_{e}, \delta_{r} \leq 0.3491$ and the thrust as $0 \leq$ $T \leq 250 \mathrm{~N}$. The UAV has the following initial conditions: $\boldsymbol{\omega}_{n, b}^{b}(0)=\left[\begin{array}{lll}0.1 & -0.2 & 0\end{array}\right]^{\top}, \mathbf{q}_{n, b}(0)=\left[\begin{array}{llll}0 & 0 & 0 & 1\end{array}\right]^{\top}$, $\mathbf{v}^{b}(0)=\left[\begin{array}{lll}25 & 0 & 0\end{array}\right]^{\top}$.

\section{A. Gain Selection}

For the command-filtered backstepping approach, the gains are chosen as $\mathbf{K}_{1}=\operatorname{diag}\{0.5,0.5,2\}, \mathbf{K}_{2}=8 \mathbf{I}$ and $\mathbf{K}_{3}=$ $\operatorname{diag}\{2,20,20\}$. The filter for the middle loop is designed as $\omega_{n}=2, \zeta=1$, with rate saturation limit of 100 , and magnitude saturation as $\frac{\pi}{2}$. The inner loop filter has both magnitude and rate saturations limits at 10 with $\omega_{n}=20$, $\zeta=1$. The NDI controller has the gains as $k_{\theta}=2, k_{\omega}=10$ and $k_{p}=2$, the decoupled method has the gains $k_{q}=20$, $\mathbf{K}_{z}=10 \mathbf{J}$ and $k_{p}=2$, and for the decoupled method with a reference the gains are chosen as $k_{1}=k_{2}=k_{3}=10$, $\mathbf{K}_{4}=10 \mathbf{J}$ and $k_{p}=2$.

The gains have been tuned empirically to obtain the best performance for each method, but there might exist better sets of gains that can improve the performance of the different methods. In general, it is more difficult to properly tune the gains of the command-filtered backstepping approach, as timescale separation must be enforced through the gains. This also puts limits on the available gains that can be used when implementing the control law and required substantial trial and error before finding the best gains for the different simulation scenarios.

When tuning the decoupled controller, it is sufficient to set the gains to a positive number that can be increased until you obtain your desired performance. When using the decoupled approach using a reference, it is recommended to choose equal gains, as that has given the best performance.

\section{B. Simulation with Perfect Knowledge}

Before "muddying the water" by including noise and model imperfections, consider the case where all controllers have perfect knowledge of the states and model. The objective is to perform a $180^{\circ}$ maneuver and make the wind frame become aligned with the NED frame. The desired states are defined as $\mathbf{q}_{n, d}=\left[\begin{array}{cccc}1 & 0 & 0 & 0\end{array}\right]^{\top}, \boldsymbol{\omega}_{n, d}^{d}=\dot{\boldsymbol{\omega}}_{n, d}^{d}=\mathbf{0}, \dot{V}_{d}=0$ and $V_{d}=40 \mathrm{~m} / \mathrm{s}$; while the wind vector is set to a constant $\mathbf{w}^{n}=\left[\begin{array}{lll}10 & 0 & 0\end{array}\right]^{\top}$.

Fig. 5 shows the rotational errors of the different control structures. It can be observed that all four methods are able to 
TABLE I

TIME TO CONVERGENCE

\begin{tabular}{ll} 
Controller & Time (s) \\
\hline Decoupled Method with Reference & 4.84 \\
Decoupled Method & 5.82 \\
Command-Filtered Backstepping & 10.73 \\
Nonlinear Dynamic Inversion & 11.77 \\
\hline
\end{tabular}

make the errors go to zero. The NDI approach makes the rudder go into saturation, thereby limiting the convergence speed. There are also some oscillations for the angular velocities. The decoupled approach exploits both the rudder and aileron (due to gain selection) and is therefore able to achieve fast convergence for both the angles and angular velocities. The command-filtered backstepping approach uses the bank angle as a control variable, and applies a rolling motion to perform the maneuver. The decoupled approach using a reference, is able to achieve the fastest convergence in less than 5 seconds, by exploiting all three actuators.

Fig. 6 shows the speed errors. All speed errors go fast to zero, but note that the command-filtered backstepping approach and the NDI have some undesired motion before convergence. Since the speed controller for the NDI is the same as for the decoupled methods, this is related to the rotational dynamics of the two controllers.

In order to have a comparative metric on the different approaches, consider the following objective function

$$
W_{1}=\boldsymbol{\Theta}_{d, w}^{\top} \boldsymbol{\Theta}_{d, w}+\left(\boldsymbol{\omega}_{n, b}^{b}\right)^{\top} \boldsymbol{\omega}_{n, b}^{b}+\tilde{V}^{2},
$$

where the quaternion-based controllers can be represented using Euler angles as

$$
\boldsymbol{\Theta}_{d, w}=\left[\begin{array}{c}
\left.\operatorname{atan} 2\left(2\left(\eta \epsilon_{1}+\epsilon_{2} \epsilon_{3}\right), 1-2\left(\epsilon_{1}^{2}+\epsilon_{2}^{2}\right)\right)\right) \\
\sin ^{-1}\left(2\left(\eta \epsilon_{2}-\epsilon_{3} \epsilon_{1}\right)\right) \\
\operatorname{atan} 2\left(2\left(\eta \epsilon_{3}+\epsilon_{1} \epsilon_{2}\right), 1-2\left(\epsilon_{2}^{2}+\epsilon_{3}^{2}\right)\right)
\end{array}\right]
$$

where $\mathbf{q}_{d, w}:=\left[\begin{array}{llll}\eta & \epsilon_{1} & \epsilon_{2} & \epsilon_{3}\end{array}\right]^{\top}$.

Fig. 7 shows a comparison between the different controllers using the metric $W_{1}$, where the decoupled method using a reference is able to achieve the best performance, followed closely by the decoupled method without a reference. Table I shows the time to convergence for the different controllers. The error is defined to have converged when $W_{1}$ crosses below $1 \cdot 10^{-3}$. It is observed that the decoupled method using a reference is $55 \%$ faster than the command-filtered backstepping approach and $59 \%$ faster than the NDI approach. Also note that the decoupled method without a reference is able to obtain very good performance, but about 1 second is saved by using the reference. One of the main reasons for the good performance of the decoupled methods are the selection of $\mathbf{K}_{z}=10 \mathbf{J}$, where $\mathbf{J}$ allows the mapping of rudder commands to the ailerons and vice versa. Using this kind of gain for the command-filtered backstepping approach did not improve the performance. For the NDI approach, a convergence time of about 6 seconds was achievable, but when introducing noise, the performance was severely deteriorated.

\section{Simulation with Noise}

One of the main advantages of the decoupled structure is that the gains can be chosen almost arbitrarily, and they are not required to enforce time-scale separation of different control loops. Since noise becomes amplified with high gains, the following simulation has included noise to see how each of the methods cope with uncertainties in the sensor-measurements. It is assumed that the inertial navigation system provides estimates of the Euler angles, quaternion and angular velocity, while a 5-hole probe provides estimates of the airspeed and angles of attack. This means that the available signals are $\boldsymbol{\Theta}_{n, b}, \mathbf{q}_{n, b}, \boldsymbol{\omega}_{n, b}^{b}, V_{a}, \alpha, \beta$, where each term is exposed to noise to model state estimation based on noisy sensor measurements (accelerometer, gyro, magnetometer, GPS and 5-hole probe).

Fig. 8 shows the simulation results. All errors converge close to zero, but it can be observed that the angles and angular velocities for the command-filtered backstepping approach oscillates much more than the other methods. Also note that there is an undesirable initial oscillation the first second for the command-filtered approach. The control signals for the different methods also contain noise due to the measurement errors, where the amplitude is higher for the NDI and commandfiltered backstepping approach than the decoupled methods. This indicates that the decoupled methods are more robust than by using time-scale separation.

\section{Simulation with Aggressive Maneuvers and Dryden Gust Model and Wind Shear Model}

To really see the performance of each method, consider the case of a more aggressive maneuver, where the aircraft must follow a time-varying trajectory described by a desired course and flight path angle. In this scenario, the Dryden gust model and wind shears ( $c f$. [32]) are also modeled to enable a more realistic simulation for studying the different controllers. The Dryden gust model and wind shear model produce a noisy wind vector as well as angular velocity components affecting the aircraft. This is an important step, as the wind variations will excite both the slow and fast modes. In order to get the best results from the command-filtered backstepping approach, its gains were changed to $\mathbf{K}_{1}=\operatorname{diag}\{0.5,0.5,2\}$, $\mathbf{K}_{2}=\operatorname{diag}\{2,8,8\}$ and $\mathbf{K}_{3}=20 \mathbf{I}$, and the rate saturation for the outer filter is reduced to 0.5 . This selection of gains is also more natural than in the previous simulations, as the gains should in general be $\left\|\mathbf{K}_{1}\right\|<\left\|\mathbf{K}_{2}\right\|<\left\|\mathbf{K}_{3}\right\|$.

Fig. 9 shows the performance of the different methods. The NDI controller is able to track the trajectory, but oscillates somewhat around the desired trajectory. The decoupled method and decoupled method with reference obtain comparable results, both having a small deviation around 25 seconds as the rudder goes into saturation. The command-filtered backstepping controller is struggling to track the trajectory, even though considerable time was spent on tuning the gains. Especially note that only a little actuation is applied by the controller, such that by increasing the saturation limitations for the filters, the convergence time of the tracking errors will most likely improve. At around 60 seconds, the flight path angle becomes negative increasing the airspeed as the 

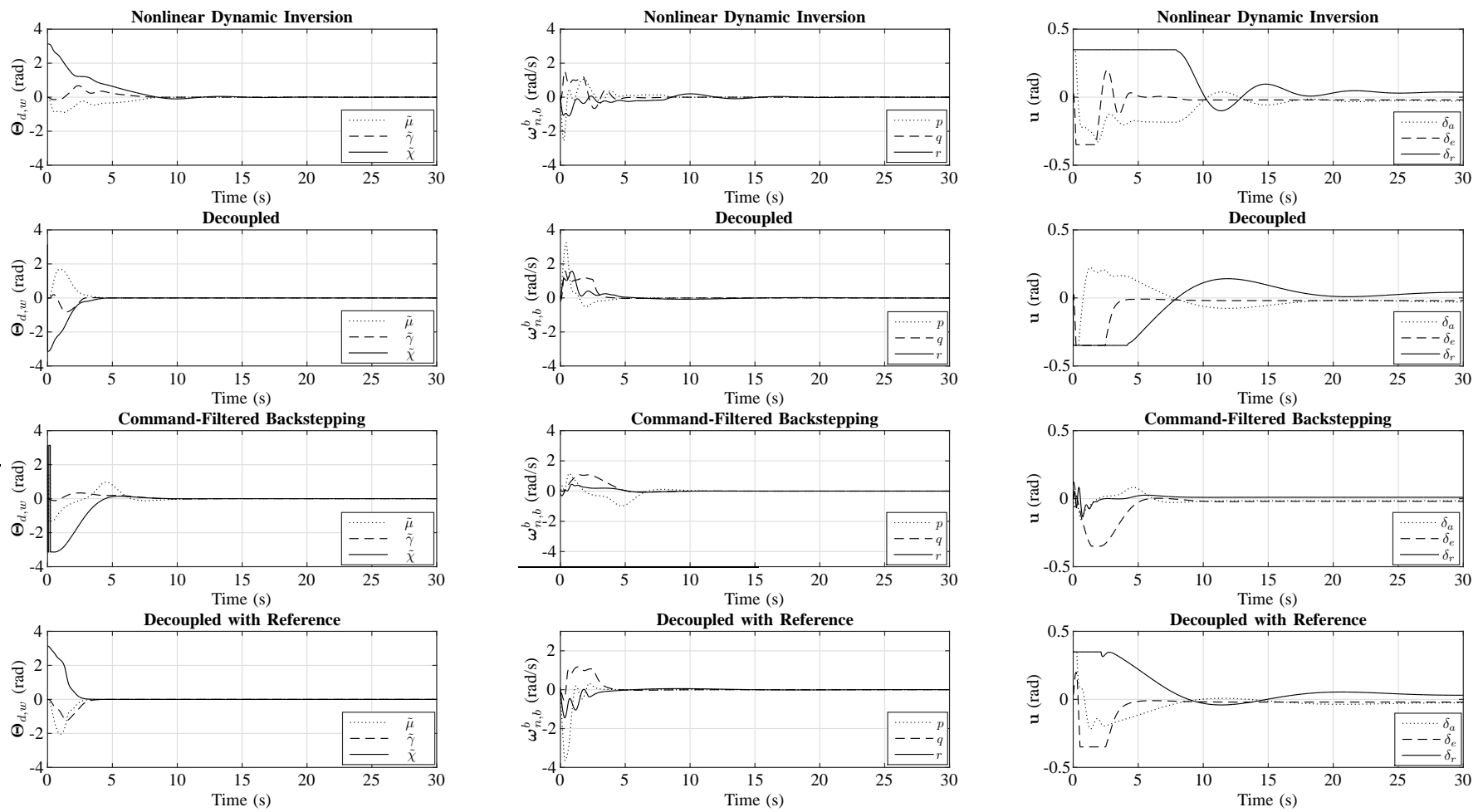

Fig. 5. Rotational tracking errors of the different control structures in the case of perfect knowledge.
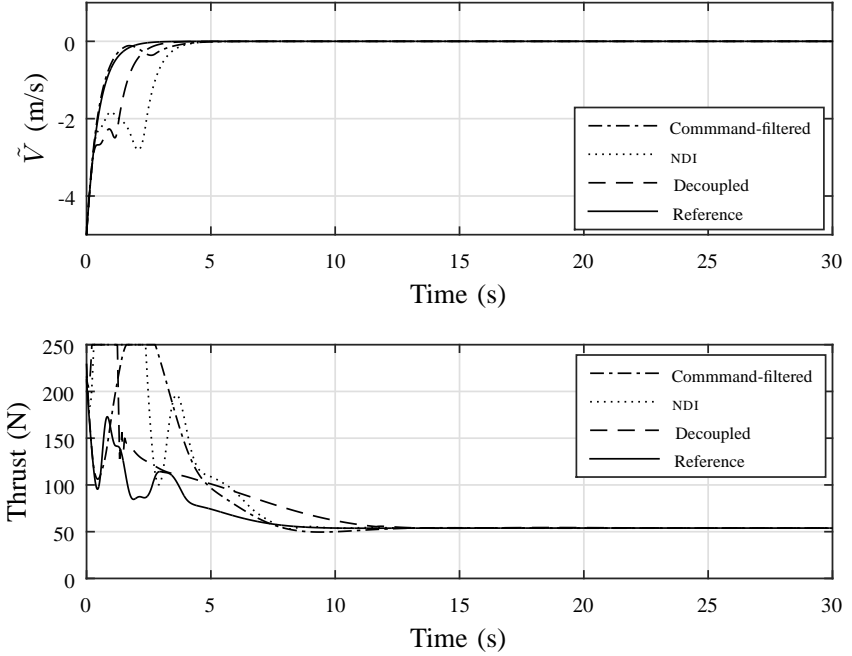

Fig. 6. Speed error and thrust for the different control structures in the case of perfect knowledge.

aircraft moves towards the Earth. This can be observed in Fig. 10 and affects the performance of each method, as less deflection is required to create a desired moment. The integral of the tracking error, $W_{2}=\int_{0}^{t}\left(\left(\chi-\chi_{d}\right)^{2}+\left(\gamma-\gamma_{d}\right)^{2}\right) d t$, is given in Fig. 12, showing that the decoupled method using the reference is able to achieve the best tracking performance.

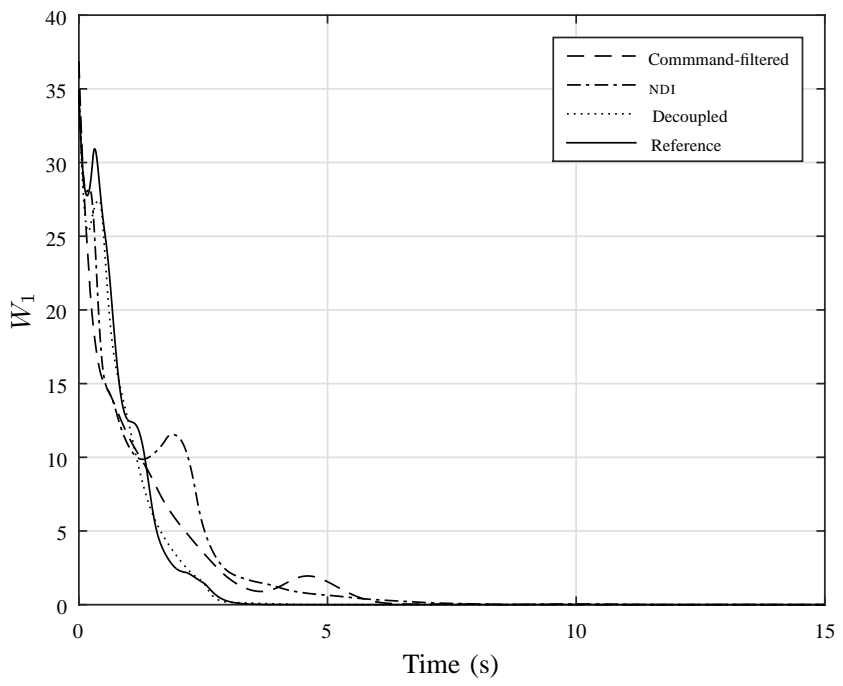

Fig. 7. Cost function showing the convergence of the different methods in the case of perfect knowledge.

\section{E. Simulation with Aggressive Maneuvers and Modeling Im- perfections}

So far, the model has been assumed to be perfectly known. In this simulation, the aerodynamic coefficients are changed to $45 \%$ of their true values, while a bias term $\mathbf{b}^{b}=\mathbf{J}^{-1}\left[\begin{array}{lll}1.3 & 1.3 & 1.3\end{array}\right]^{\top}$ is added to the angular acceleration (12). Trying to introduce larger errors in the aerodynamics 

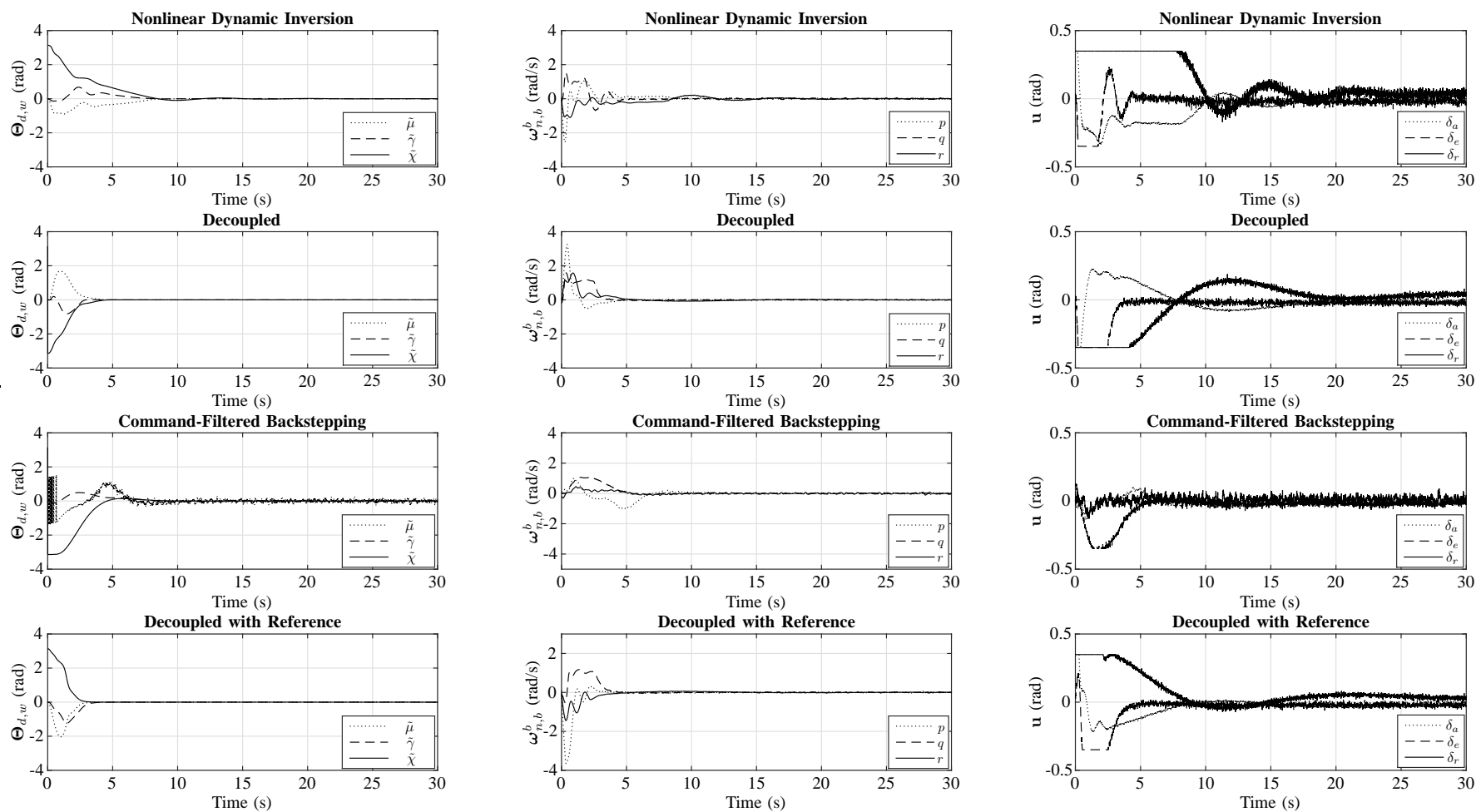

Fig. 8. Comparison between the different control structures when including noise.
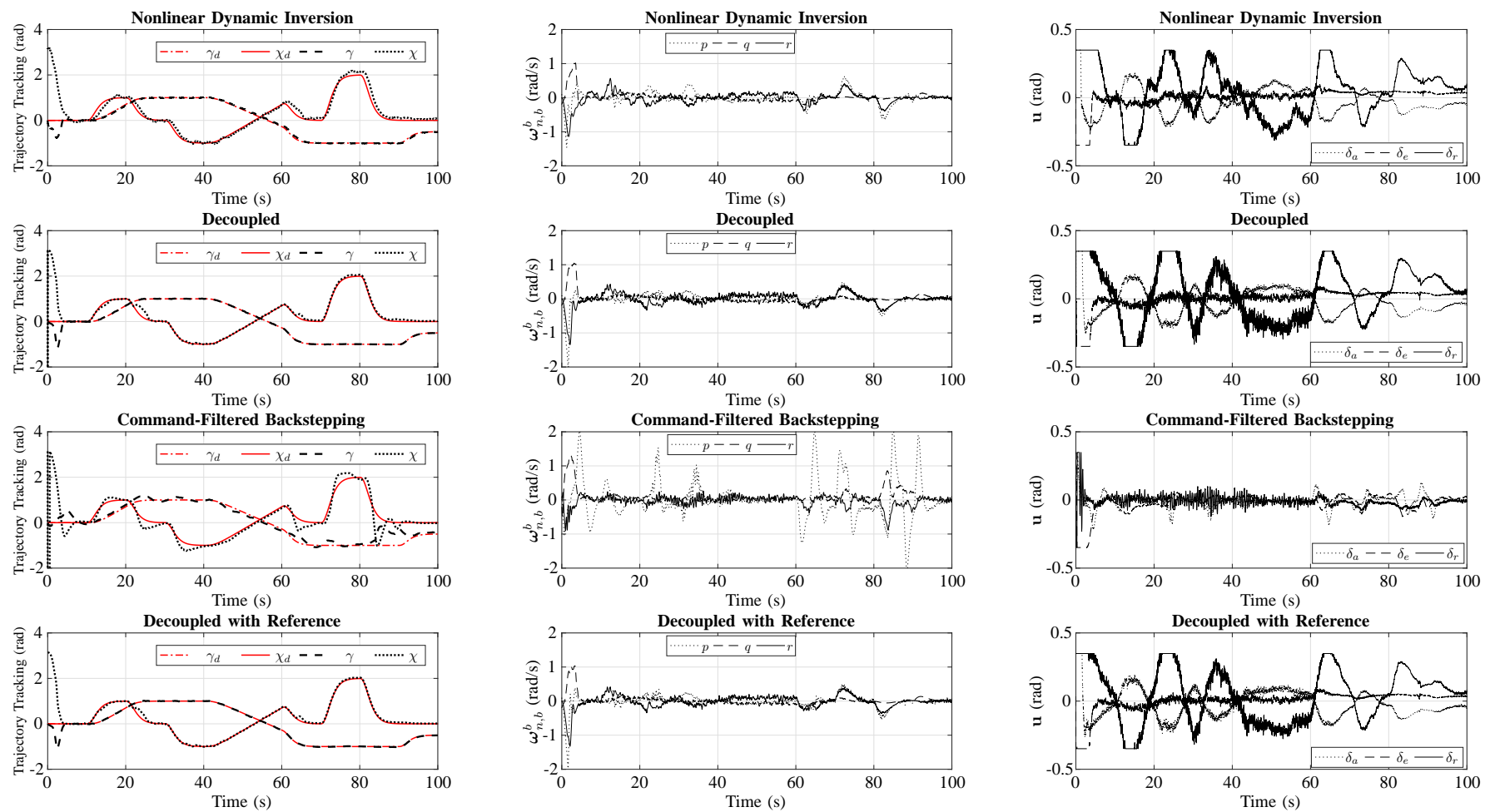

Fig. 9. Comparison between the different control structures when performing tracking of a more challenging desired flight path and course angles. Note that at around 60 seconds, the thruster goes to zero making due to the negative flight path angle making the airspeed increase to about $80 \mathrm{~m} / \mathrm{s}$ (airbrakes are not modeled). 

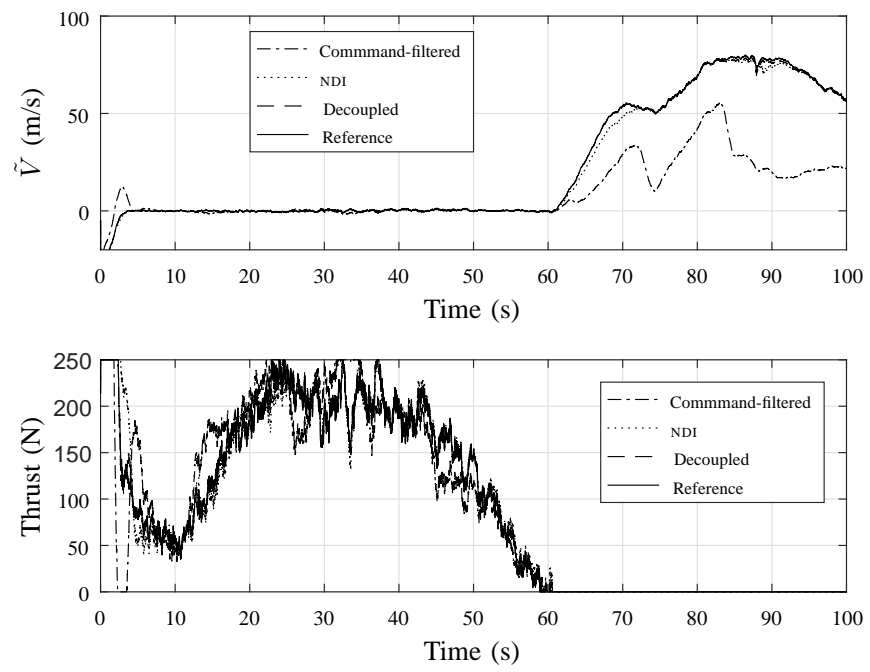

Fig. 10. Airspeed error and thrust for the different control structures when tracking an aggressive trajectory. Note that airbrakes are not used, such that the speed increases when the aircraft is moving with a negative flight path angle and zero thrust.

or bias term resulted in a loss of control when using the command-filtered backstepping approach.

Fig. 11 shows the performance of the different controllers, where all controllers are more or less able to track the trajectory, but the NDI and command-filtered backstepping controllers have a higher inaccuracy than the decoupled methods. Further, a comparison of the impact of modeling errors is shown in Fig. 12 showing the integral of the course and flight path errors. It follows that the command-filtered backstepping approach is most sensitive to model imperfections. One interesting aspect with this comparison, is the fact that the decoupled method without a reference is able to achieve the best performance. This is due to the bias term, which will be a good or bad term dependent on the direction of rotation. As can be seen in Fig. 11, the decoupled method rotates initially in an opposite direction compared to the other solutions in this simulation, and as such gets help from the bias term.

\section{DISCUSSION}

The main limitation for the convergence time of the command-filtered backstepping is the static limitations in the command filters. This is natural, as the filters impose saturation to the signals, and thereby limits the overall performance. A future direction with regards to this is to apply nonlinear limitations to these filters, as it is desirable to obtain large commands when the error is large, and small commands when the error is small while keeping the system stable. The work by [33] on exponential gain selection can be a good starting point for implementing such a solution in the filters. This will probably improve the performance of the command-filtered backstepping approach. Another issue with the commandfiltered backstepping approach that became evident through simulations, is that the bank and roll angles do not go to zero. This is due to the method of finding the desired bank angle from the non-affine form, resulting in a non-zero desired angle.
All the controllers presented in this paper should be implementable on a standard microcontroller, e.g. a STM32f4 or similar chipset running a real-time operating system. The easiest controller to implement from these is the NDI, as it has a very simple structure; while the decoupled methods and command-filtered backstepping approach will require more time to implement, but should still run fine on any modern microcontroller.

While this work has mainly focused on classical NDI and command-filtered backstepping approaches with their inherent time-scale separation issues, it is important to stress the fact that there are adaptive versions of these controllers [34], [35] and [36]. The adaptive controllers will naturally exhibit very different performance in the presence of modeling imperfections, but adaptive control also allows for dealing with the time-scale separation issues, and as such can be considered an important step to improve the performance of any of the controllers presented in this paper.

\section{CONCLUSION}

This paper has presented three different control structures, where the different simulations show that the new decoupled method using a reference signal shows very good performance when compared to nonlinear dynamic inversion and the command-filtered backstepping approach. By increasing the gains sufficiently, the convergence time can be made very short, with relatively small steady-state error due to the noise or modeling imperfections. While the new approach has advantages over the existing approaches, it is important to note that the NDI and command-filtered backstepping can increase their gains to most likely provide comparable results with the decoupled method, but it is challenging to properly tune the gains due to the inner-middle-outer loop structures.

\section{ACKNOWLEDGEMENT}

The authors would like to thank the reviewers and editor for constructive feedbacks that helped improve the paper.

\section{APPENDIX A}

The YF-22 UAV model is given in [17] and is reproduced here. Note that the stabilizer is treated as an elevator.

$\begin{array}{lll}m=20.64 & J_{x x}=1.607 & J_{y y}=7.51 \\ J_{z z}=7.18 & J_{x z}=-0.59 & b=1.96 \\ \bar{c}=0.76 & S=1.37 & C_{D_{0}}=0.008 \\ C_{D_{\alpha}}=0.508 & C_{D_{q}}=0 & C_{D_{\delta_{e}}}=-0.034 \\ C_{L_{0}}=-0.049 & C_{L_{\alpha}}=3.258 & C_{L_{q}}=0 \\ C_{L_{\delta_{e}}}=0.189 & C_{m_{0}}=0.022 & C_{m_{\alpha}}=-0.473 \\ C_{m_{q}}=-3.449 & C_{m_{\delta_{e}}}=-0.364 & C_{Y_{0}}=0.015 \\ C_{Y_{\beta}}=0.272 & C_{Y_{p}}=1.215 & C_{Y_{r}}=-1.161 \\ C_{Y_{\delta_{a}}}=0.183 & C_{Y_{\delta_{r}}}=-0.459 & C_{l_{0}}=-0.001 \\ C_{l_{\beta}}=-0.038 & C_{l_{p}}=-0.213 & C_{l_{r}}=0.114 \\ C_{l_{\delta_{a}}}=-0.056 & C_{l_{\delta_{r}}}=0.014 & C_{n_{0}}=0 \\ C_{n_{\beta}}=0.036 & C_{n_{p}}=-0.151 & C_{n_{r}}=-0.195 \\ C_{n_{\delta_{a}}}=-0.036 & C_{n_{\delta_{r}}}=-0.055 & \end{array}$



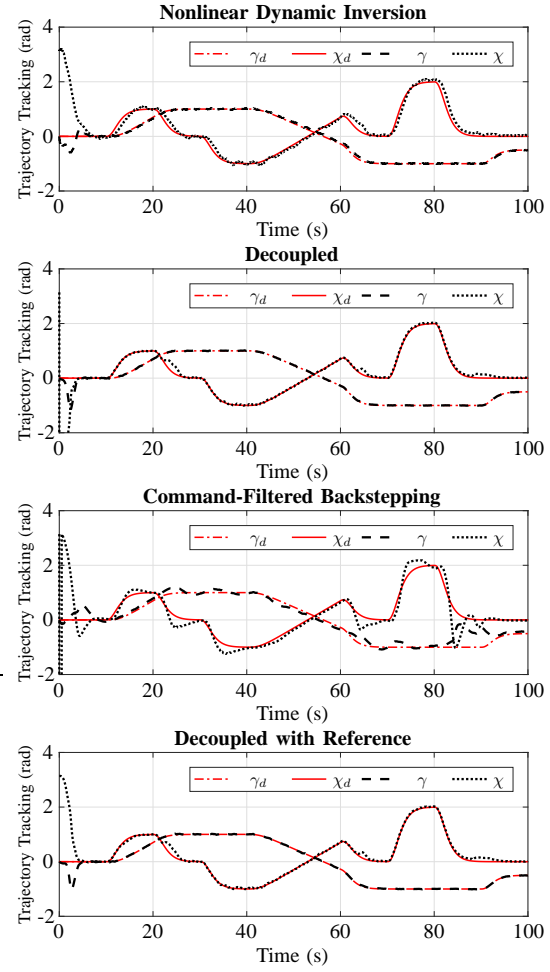
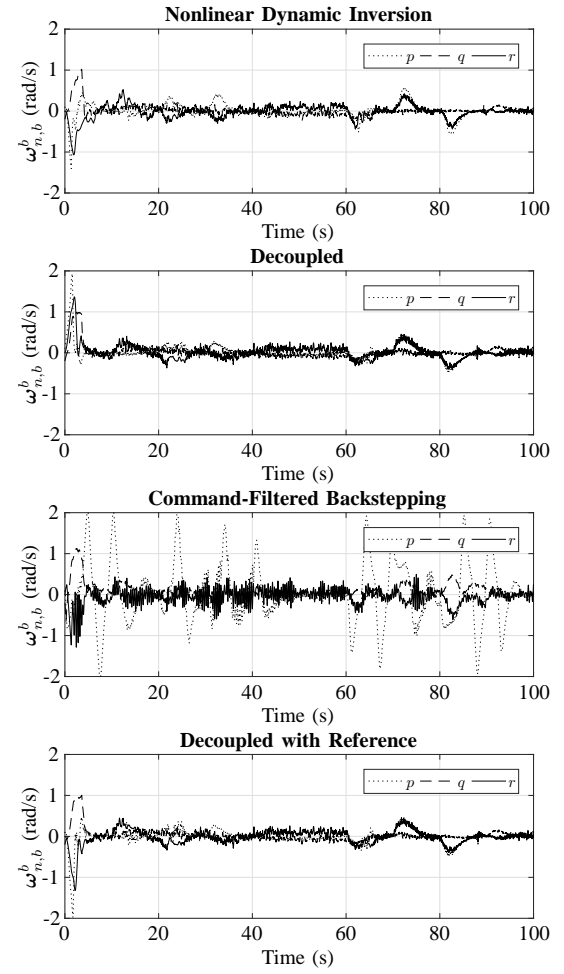
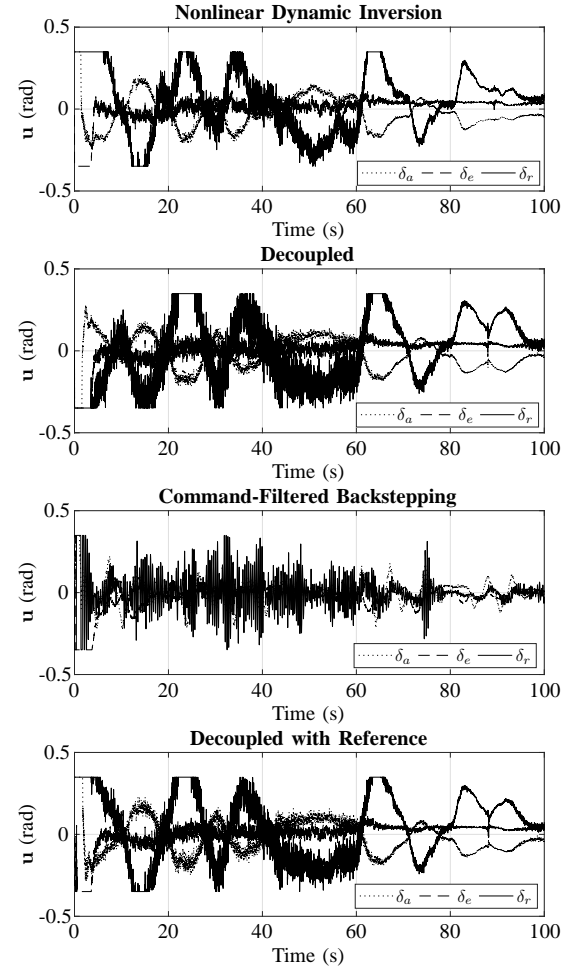

Fig. 11. Comparison between the different control structures when performing trajectory tracking in the presence of modeling imperfections.
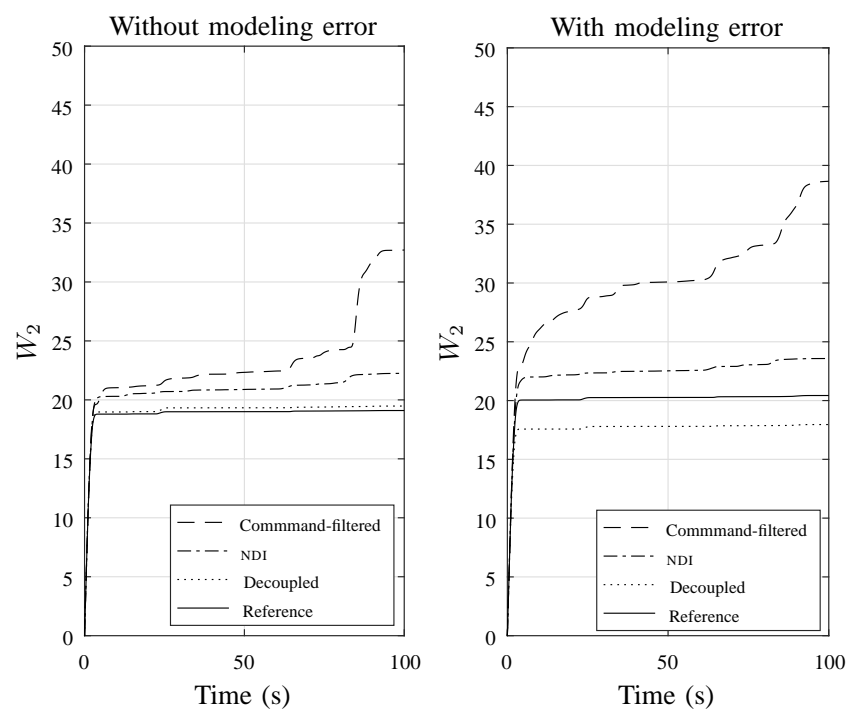

Fig. 12. Showing the integral of the tracking errors over time when following the aggressive trajectory.

\section{REFERENCES}

[1] W. Ren and R. W. Beard, "Trajectory tracking for unmanned air vehicles with velocity and heading rate constraints," IEEE Transactions on Control Systems Technology, vol. 12, no. 5, pp. 706-716, 2004.

[2] R. A. Nichols, R. T. Reichert, and W. J. Rugh, "Gain scheduling for h-infinity controllers: A flight control example," IEEE Transactions on Control Systems Technology, vol. 1, no. 2, pp. 69-79, 1993.

[3] N. Slegers, J. Kyle, and M. Costello, "Nonlinear model predictive control technique for unmanned air vehicles," Journal of Guidance, Control and Dynamics, vol. 29, no. 5, pp. 1179-1188, 2006.

[4] S. H. Lane and R. F. Stengel, "Flight control design using non-linear inverse dynamics," Automatica, vol. 24, no. 4, pp. 471-483, 1988.

[5] S. A. Snell, D. F. Enns, and W. L. Garrard, "Nonlinear inversion flight control for a supermaneuverable aircraft," Journal of Guidance, Control, and Dynamics, vol. 15, no. 4, pp. 976-984, 1992.

[6] Q. Wang and R. F. Stengel, "Robust nonlinear flight control of a high performance aircraft," IEEE Transactions on Control Systems Technology, vol. 13, no. 1, pp. 15-26, 2005.

[7] T. Lee and Y. Kim, "Nonlinear adaptive flight control using backstepping and neural networks controller," Journal of Guidance, Control and Dynamics, vol. 24, no. 4, pp. 675-682, 2001.

[8] J. W. C. Robinson, "Block backstepping for nonlinear flight control law design," in Nonlinear Analysis and Synthesis Techniques for Aircraft Control (D. Bates and M. Hagström, eds.), Springerlink, ISBN: 978-3540-73718-6, 2007.

[9] J. Farrell, M. Sharma, and M. Polycarpou, "Backstepping-based flight control with adaptive function approximation," Journal of Guidance, Control, and Dynamics, vol. 28, no. 6, pp. 1089-1101, 2005.

[10] L. Sonneveldt, E. R. van Oort, Q. P. Chu, and J. A. Mulder, "Nonlinear adaptive trajectory control applied to an F-16 model," Journal of Guidance, Control and Dynamics, vol. 32, no. 1, pp. 25-39, 2009.

[11] E. Oland and R. Kristiansen, "A decoupled approach for flight control," Modeling, Identification and Control, vol. 37, no. 4, pp. 237-246, 2016.

[12] E. Johnson and A. J. Calise, "Pseudo-control hedging: a new method for adaptive control," in Proceedings of the Workshop on Advances in Guidance and Control Technology, (Redstone Arsenal, Alabama, USA), 2000.

[13] E. Oland, T. S. Andersen, and R. Kristiansen, "Subsumption architecture applied to flight control using composite rotations," Automatica, vol. 69, pp. 195-200, 2016.

[14] R. F. Stengel, Flight dynamics. Princeton University Press, ISBN 0-69111407-2, 2004.

[15] B. L. Stevens and F. L. Lewis, Aircraft control and simulation. 2nd ed., Wiley, ISBN 978-0-471-37145-8, 2003.

[16] B. Etkin, Dynamics of atmospheric flight. Dover Publications, Inc., ISBN 0-486-44522-4, 1972. 
[17] G. Campa, Y. Gu, B. Seanor, M. R. Napolitano, L. Pollini, and M. L. Fravolini, "Design and flight-testing of non-linear formation control laws," Control Engineering Practice, vol. 15, pp. 1077-1092, 2007.

[18] O. Egeland and J. T. Gravdahl, Modeling and simulation for automatic control. Marine Cybernetics, Trondheim, Norway, ISBN 82-92356-01-0, 2002.

[19] Honeywell and Lockheed Martin, "Application of multivariable control theory to aircraft control laws. final report: multivariable control design guidelines," tech. rep., Honeywell TC. and Lockheed Martin Skunk Works and Lockheed Martin TAS., 1996.

[20] D. Ito, J. Georgie, J. Valasek, and D. T. Ward, "Reentry vehicle flight controls design guidelines: dynamic inversion," tech. rep., Flight Simulation Laboratory, Texas Engineering Experiment Station, Texas A\&M University, NASA, 2002

[21] M. Breivik and T. I. Fossen, "Guidance-based path following for autonomous underwater vehicles," in Proceedings of the MTS/IEEE OCEANS, (Washington, DC, USA), 2005.

[22] A. Levant, "Robust exact differentiation via sliding mode technique," Automatica, vol. 34, no. 3, pp. 379-384, 1998

[23] T. I. Fossen, Handbook of marine craft hydrodynamics and motion control. John Wiley \& Sons Ltd., ISBN: 978-1-119-99149-6, 2011.

[24] E. Oland and R. Kristiansen, "Quaternion based attitude control for a fixed-wing UAV using backstepping," in Proceedings of the 34th IEEE Aerospace Conference, (Big Sky, MT, USA), 2013.

[25] R. Kristiansen, A. Loría, A. Chaillet, and P. J. Nicklasson, "Spacecraft relative rotation tracking without angular velocity measurements," $\mathrm{Au}$ tomatica, vol. 45, no. 3, pp. 750-756, 2009.

[26] S. P. Bhat and D. S. Bernstein, "A topological obstruction to continuous global stabilization of rotational motion and the unwinding phenomenon," Systems \& Control Letters, vol. 39, pp. 63-70, 2000

[27] R. Schlanbusch, A. Loría, and P. J. Nicklasson, "On the stability and stabilization of quaternion equilibria of rigid bodies," Automatica, vol. 48, no. 12, pp. 3135-3141, 2012.

[28] H. K. Khalil, Nonlinear systems. 3rd ed., Prentice Hall, ISBN 0-13067389-7, 2002

[29] M. D. Tandale and J. Valasek, "Adaptive dynamic inversion control with actuator saturation constraints applied to tracking spacecraft maneuvers," The Journal of Astronautical Sciences, vol. 52, no. 4, pp. 517-530, 2005.

[30] E. Oland and R. Kristiansen, "Adaptive flight control with constrained actuation," in Proceedings of the American Control Conference, (Portland, OR, USA), 2014.

[31] E. Oland, T. S. Andersen, and R. Kristiansen, "Actuator desaturation for a fixed-wing UAV using speed modification," in Proceedings of the ICNPAA Congress on Mathematical problems in engineering, aerospace and sciences, (Narvik, Norway), 2014.

[32] S. H. Pourtakdoust, M. Kiani, and A. Hassanpour, "Optimal trajectory planning for flight through microburst wind shears," Aerospace Science and Technology, vol. 15, no. 7, pp. 567-576, 2011.

[33] R. Schlanbusch and R. Kristiansen, "Control of spacecraft formation with disturbance rejection and exponential gains," Modeling, Identification and Control, vol. 34, no. 1, pp. 11-18, 2013

[34] N. Hovakimyan, E. Lavretsky, and C. Cao, "Adaptive dynamic inversion via time-scale separation," IEEE Transactions on Neural Networks, vol. 19, no. 10, pp. $1702-1711,2008$.

[35] C. Li, W. Jing, and C. Gao, "Adaptive backstepping-based flight control system using integral filters," Aerospace Science and Technology, vol. 13, no. 2-3, pp. 105-113, 2009.

[36] L. Sonneveldt, Adaptive backstepping flight control for modern fighter aircraft. $\mathrm{PhD}$ thesis, Delft University of Technology, Delft, The Netherlands, 2010.

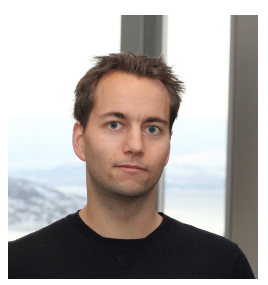

Espen Oland was born in Arendal, Norway in 1981 He completed his master's degree in Space Technology in 2008 at Narvik University College (NUC) and worked as an Assistant Professor at NUC from 2008-2010 where he contributed to the design and completion of the HiNCube student satellite where he has had the main responsibility for the attitude determination and control system. From September to December 2012 he was a visiting research scholar at Ohio State University under the supervision of Andrea Serrani. He received a Ph.D. in Engineering Cybernetics from NTNU in 2014 with the thesis "Nonlinear Control of Fixed-Wing Unmanned Aerial Vehicles" under the supervision of Raymond Kristiansen and Jan Tommy Gravdahl. From 2014 to 2017 he worked as a researcher at the research institute Teknova and is currently working as a control system manager at Kitemill AS and as an Associate Professor in the Department of Electrical Engineering at UiT - The Arctic University of Norway. His research interests include control of: unmanned aerial vehicles, airborne wind energy systems, spacecraft, underactuated rigid bodies, and behavioral control methods.

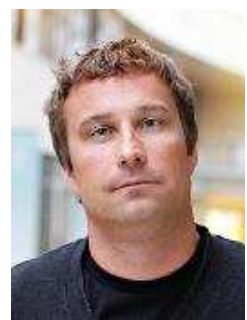

Raymond Kristiansen was born in Finnsnes, Norway, in 1977. He received his B.Sc. degree in Computer Science from Narvik University College (NUC), Norway, in 1998, and his M.Sc. degree in Engineering Cybernetics from the Norwegian University of Science \& Technology (NTNU), Norway, in 2000. From September through November 2005 he was a graduate scholar visitor at the "Laboratoire de Signaux et Systemes", Supelec in Paris, France, under the supervision of Dr. Antonio Loria. He received his Ph.D. degree in Engineering Cybernetics from NTNU in 2008 with the thesis "Dynamic Synchronization of Spacecraft: Modeling and Coordinated Control of Leader-Follower Spacecraft Formations" under supervision of Professor Per Johan Nicklasson at NUC and Professor Jan Tommy Gravdahl at NTNU. R. Kristiansen is currently a Professor at the Department of Electrical Engineering at UiT - The Arctic University of Norway. He is the (co)author of more than 40 scientific publications in international journals and conference proceedings, and is a member of the editorial board of the Elsevier Journal Simulation Modelling Practice and Theory. His research interests include modeling and nonlinear control of aerospace systems in general, and spacecraft and UAVs in particular, with a special focus on synchronization and coordinated control in formations.

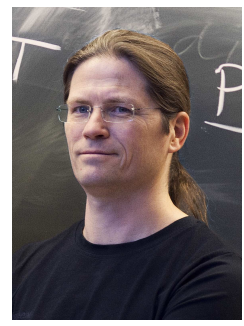

Jan Tommy Gravdahl is professor at the Norwegian University of Science and Technology (NTNU). He was born in 1969 and graduated siv.ing (1994) and dr.ing (1998) in Engineering Cybernetics, NTNU. From 1998 to 2001 he was a postdoctoral researcher with the Department of Engineering $\mathrm{Cy}$ bernetics. He was appointed Associate Professor (2001) and Professor (2005) at the same department, where he served as deputy department head 200607 and department head in 2008/09. In 2007/08 he was with The Centre for Complex Dynamic Systems and Control (CDSC), The University of Newcastle, Australia. His current research interests include mathematical modeling and nonlinear control in general and with application to turbomachinery, spacecraft, robots, ships and nanopositioning devices. He has supervised the graduation of $80 \mathrm{MSc}$ and $10 \mathrm{PhD}$. He has published more than 150 papers at conferences and in international journals and in 2000 he received the IEEE Transactions on Control Systems Technology Outstanding Paper Award. He is author of Compressor Surge and Rotating Stall: Modeling and Control (Springer 1999), co-author of Modeling and Simulation for Automatic Control (Marine Cybernetics 2002), Snake Robots: Modeling, Mechatronics, and Control (Springer 2013), Vehicle-Manipulator Systems: Modeling for Simulation, Analysis and Control (Springer 2014) and co-editor of Group Coordination and Cooperative Control (Springer 2006). 